\title{
Gaia DR2 giants in the Galactic dust - II. Application of the reddening maps and models.
}

\author{
George A. Gontcharov ${ }^{\star}$ and Aleksandr V. Mosenkov \\ Central Astronomical Observatory, Russian Academy of Sciences, 65/1 Pulkovskoye chaussee, St. Petersburg, 196140 Russia
}

Accepted 2020 August 31; Received 2020 August 23; in original form 2020 April 9

\begin{abstract}
We exploit a complete sample of 101810 Gaia DR2 giants, selected in Paper I in the space cylinder with a radius of $700 \mathrm{pc}$ around the Sun and a height of $|Z|=1800 \mathrm{pc}$, using the Gaia DR2 parallaxes, $G_{\mathrm{BP}}$ and $G_{\mathrm{RP}}$ photometry, and WISE W3 photometry. We explain the spatial variations of the modes of the observables $G_{\mathrm{BP}}-G_{\mathrm{RP}}$ and $G_{\mathrm{RP}}-W 3$ by the spatial variations of the corresponding reddenings described in the GM20 3D dust distribution model. Presented in this paper, GM20 is an advanced version of the model introduced by Gontcharov in 2009. GM20 proposes two intersecting dust layers, along the Galactic mid-plane and in the Gould Belt, with exponential vertical and sinusoidal longitudinal variations of the dust spatial density in each layer. The Belt layer is an ellipse, oriented nearly between the centre and anticentre of the Galaxy, and with a semi-major and semi-minor axes of 600 and $146 \mathrm{pc}$, respectively. $G_{\mathrm{BP}}-G_{\mathrm{RP}}$ and $G_{\mathrm{RP}}-W 3$ give similar solutions, but different equatorial layer scale heights of $150 \pm 15$ and $180 \pm 15 \mathrm{pc}$, respectively, and $\left(G_{\mathrm{BP}}-G_{\mathrm{RP}}\right)_{0}=(1.14 \pm 0.01)-(0.022 \pm 0.010)|Z|$, $\left(G_{\mathrm{RP}}-W 3\right)_{0}=(1.44 \pm 0.01)-(0.015 \pm 0.010)|Z|$, where $Z$ is in kpc. We compare GM20 with several 3D reddening models and maps in their ability to predict the observed colour modes. GM20 and the 3D map by Gontcharov appear to be the best among the models and maps, respectively. However, the most reliable models and maps mainly disagree only in their estimates of low reddening, including the reddening across the whole dust layer.
\end{abstract}

Key words: stars: late-type - dust, extinction - ISM: individual objects: Gould Belt - ISM: structure - local interstellar matter - solar neighbourhood

\section{INTRODUCTION}

In our previous paper (Gontcharov \& Mosenkov 2020, hereafter Paper I) we verified the usage of the parallaxes and photometry from Gaia DR2 (Gaia Collaboration 2018a,b) in order to simultaneously derive some key properties of the Galactic dust layer and clump giants embedded into or seen through this layer.

In Paper I we selected a complete sample of 101810 Gaia DR2 giants in the giant clump domain of the Hertzsprung-Russell (HR) diagram within the space cylinder with a radius of $700 \mathrm{pc}$ around the Sun and a height of $|Z|=1800 \mathrm{pc}$ along the $Z$ Galactic rectangular coordinate. To select the sample, we used the Gaia DR2 parallaxes in combination with the photometry from the Gaia DR2 $G_{\mathrm{BP}}, G_{\mathrm{RP}}$ bands and the $W 3$ band from Wide-field Infrared Survey Explorer (WISE, allWISE, Wright et al. 2010).

Gontcharov (2017b) had shown that the modes of the intrinsic (dereddened) colours and absolute magnitudes of such a sample are completely defined by the clump giants ${ }^{1}$. The intrinsic colours and absolute magnitudes of the clump vary only with age and metallic-

\footnotetext{
^ E-mail: georgegontcharov@yahoo.com

1 To obtain the modes, we follow Gontcharov (2017b), who rounds the observables up to $0.01 \mathrm{mag}$ and finds the tops of their histograms in each
}

ity and can be presented in the space under consideration as some simple functions of $|Z|$ (Girardi 2016; Önal Taş et al. 2016). Therefore, we assumed for our sample that the spatial variations of the modes of the observables $G_{\mathrm{BP}}-G_{\mathrm{RP}}, G_{\mathrm{RP}}-W 3, M_{\mathrm{G}_{\mathrm{BP}}}+A_{\mathrm{G}_{\mathrm{BP}}}$, $M_{\mathrm{G}}+A_{\mathrm{G}_{\mathrm{RP}}}$, and $M_{\mathrm{W} 3}+A_{\mathrm{W} 3}$ reflect the spatial variations of the reddenings $E\left(G_{\mathrm{BP}}-G_{\mathrm{RP}}\right)$ and $E\left(G_{\mathrm{RP}}-W 3\right)$, extinctions $A_{\mathrm{G}_{\mathrm{BP}}}$, $A_{\mathrm{G}_{\mathrm{RP}}}$, and $A_{\mathrm{W} 3}$ in a combination with some linear gradients of the dereddened colours $\left(G_{\mathrm{BP}}-G_{\mathrm{RP}}\right)_{0}$ and $\left(G_{\mathrm{RP}}-W 3\right)_{0}$ and absolute magnitudes $M_{\mathrm{G}_{\mathrm{BP}}}, M_{\mathrm{G}}$, and $M_{\mathrm{W} 3}$ with $|Z|$.

Without an application of any model of dust distribution (or spatial variations of extinction/reddening), we analyzed in Paper I the spatial variations of the observables within (i) a thin coordinate layer of $|Z|<40 \mathrm{pc}$, (ii) a narrow vertical cylinder of $\left(X^{2}+Y^{2}\right)^{0.5}<$ $80 \mathrm{pc}$ (where $X$ and $Y$ are the Galactic rectangular coordinates), and (iii) a space behind the layer limited by $|Z|>400 \mathrm{pc}$ and the latitude $|b|>68.75^{\circ}$. In particular, this allowed us to obtain the following estimates of the absolute magnitudes and intrinsic colours of the clump:

$M_{\mathrm{G}_{\mathrm{BP}}}=(0.91 \pm 0.01)+(0.01 \pm 0.01)|Z|$,

spatial cell. In the case of multimodal histogram, the lowest value is selected, since it is more probable for the unreddened or slightly reddened clump. 
$M_{\mathrm{G}_{\mathrm{RP}}}=(-0.235 \pm 0.01)+(0.04 \pm 0.01)|Z|$,

$M_{\mathrm{W} 3}=(-1.67 \pm 0.01)+(0.07 \pm 0.01)|Z|$,

$\left(G_{\mathrm{BP}}-G_{\mathrm{RP}}\right)_{0}=(1.145 \pm 0.01)-(0.03 \pm 0.01)|Z|$,

$\left(G_{\mathrm{RP}}-W 3\right)_{0}=(1.435 \pm 0.01)-(0.03 \pm 0.01)|Z|$,

where $Z$ is in $\mathrm{kpc}$.

We compared these values with the theoretical predictions from PAdova and TRieste Stellar Evolution Code (PARSEC, Marigo et al. $2017^{2}$ ), MESA Isochrones and Stellar Tracks (MIST, Paxton et al. 2011, 2013; Choi et al. 2016; Dotter 2016 ${ }^{3}$ ), and the Bag of Stellar Tracks and Isochrones (IAC-BaSTI, Hidalgo et al. $2018^{4}$ ). This allowed us to estimate the clump median age and $[\mathrm{Fe} / \mathrm{H}]$ with their linear vertical gradients within $|Z|<1.7 \mathrm{kpc}$ as $(2.3 \pm 0.5)+(3.2 \pm 1.6)|Z|$ Gyr and $(-0.08 \pm 0.08)-(0.16 \pm 0.07)|Z|$, respectively, where $Z$ is expressed in kpc.

In Paper I we found the extinctions and reddenings across the whole dust half-layer below or above the Sun. They converge to the reddening $E(B-V) \approx 0.06$ mag by use of the extinction laws of Davenport et al. (2014, hereafter DIB14) for the high latitudes, Schlafly et al. (2016, hereafter SMS16), Wang \& Chen (2019, hereafter WC19), and Cardelli, Clayton \& Mathis (1989, hereafter CCM89) with $R_{\mathrm{V}} \equiv A_{\mathrm{V}} / E(B-V)=3.1$.

The aim of this paper is to use the same sample, but with a 3D dust distribution model applied, in order to simultaneously derive some properties of the Galactic dust layer and clump giants embedded into or seen through this layer.

To choose a proper 3D dust distribution model we take into account the note of Dame et al. (2001): 'most of the major local molecular clouds appear to follow Gould's Belt,. . . the apparent disk of OB stars, gas, and dust surrounding the Sun and inclined $\approx 20^{\circ}$ to the Galactic plane'. Gontcharov (2012), following Gontcharov (2009), proposed to consider two dust layers intersecting near the Sun: the equatorial one and the layer in the Gould Belt. They were described in the first (Gontcharov 2009, hereafter G09) and second (Gontcharov 2012, hereafter G12) versions of the model of the 3D dust distribution. We revise this model in the current study.

Then we verify this revised version of the model (hereafter GM20) among other dust distribution models and maps ${ }^{5}$ in their ability to correctly reproduce the modes of the colours $G_{\mathrm{BP}}-G_{\mathrm{RP}}$ and $G_{\mathrm{RP}}-W 3$ for our sample.

We should take into account some results of the previous verifications of the most advanced models and maps by Gontcharov (2017a); Gontcharov \& Mosenkov (2017a,b, 2018, 2019). They have shown that:

(i) 2D maps by Schlegel, Finkbeiner \& Davis (1998, hereafter SFD98) and Meisner \& Finkbeiner (2015, hereafter MF15), which

\footnotetext{
2 http://stev.oapd.inaf.it/cgi-bin/cmd

3 http://waps.cfa.harvard.edu/MIST/

4 http://basti-iac.oa-abruzzo.inaf.it/index.html. IAC-

BaSTI does not provide any estimate for $W 3$ band

5 A model is a representation by some formulas, while a map is a representation in a tabular view.
}

provide only a cumulative reddening to infinity, are useless in describing the distribution of the local dust.

(ii) The same is true for any 3D follower of these maps. An example is the map of Drimmel, Cabrera-Lavers \& López-Corredoira (2003, hereafter DCL03), based on the model of Drimmel \& Spergel (2001). It includes a description of a dust segment of the local arm based on some dust emission at an unknown distance taken from a 2D map. However, the source of this emission seems to be too distant. DCL03 note: 'Ideally this region about the Sun should be described by a more detailed local model of the dust distribution.'

(iii) Any incompleteness of the stellar sample in use may cause a bias in the results, usually towards low reddening. This may be a reason for some unreliable estimates with the map of Lallement et al. (2014, hereafter LVV14).

(iv) The best representations of the local dust distribution have been obtained using map of Gontcharov (2017a, hereafter G17). It is successful due to the use of unsaturated photometry for a complete sample of nearby stars. G12 has proven to be the best among the models due to a direct consideration of the Gould Belt as an important local dust container. Gontcharov \& Mosenkov (2017b, 2018) concluded that G12 deserves more attention and a further refinement. This is fulfilled in the current study.

This paper is organized as follows. In Sect. 2 we analyze the spatial variations of the colour modes in order to derive a general pattern of the 3D dust distribution and to constrain a proper geometry of the model. In Sect. 3 we consider a simplest model and GM20 to fit the observed variations of the colour modes. We compare several 3D models and maps in their ability to fit the data in Sect. 4. Some notes on the reddening across the whole dust layer are given in Sect. 5. We summarize our findings and state our conclusions in Sect. 6.

\section{THE MODES THROUGHOUT THE WHOLE SPACE}

Some information about the 3D dust distribution can be derived from mode $\left(G_{\mathrm{BP}}-G_{\mathrm{RP}}\right)$ and mode $\left(G_{\mathrm{RP}}-W 3\right)$ calculated in some cells located throughout the whole space cylinder under consideration.

As we note in Sect. 1, to obtain the modes, we round the observables up to $0.01 \mathrm{mag}$ and find the tops of their histograms in each cell. In the case of multimodal histogram, the lowest value is selected, since it is more probable for the unreddened or slightly reddened clump.

As discussed in Paper I, space cells with 400 giants are optimal to calculate the modes. This number allows us to keep a balance between precision and spatial resolution of the results. In such a cell, the uncertainty of the modes is defined by natural fluctuations of the interstellar medium between us and stars in the cell. These fluctuations are followed by related fluctuations of the extinction, reddening, and observables.

Initially, we set 101810 cells with our stars as their centres. For each cell the modes of the colours are calculated by use of the stars within a radius of

$110 /\left(\mathrm{e}^{-|Z| / 1000}\right)$,

where $Z$ is expressed in pc. The radius varies from $110 \mathrm{pc}$ at the Galactic mid-plane to $665 \mathrm{pc}$ at $|Z|=1800 \mathrm{pc}$. Yet, within the dust layer it increases slowly: to only $181 \mathrm{pc}$ at $|Z|=500 \mathrm{pc}$. Such radius allows us to use about 400 stars in each cell and calculate the colour modes with a precision at a level of $0.02 \mathrm{mag}$. Finally, in order to 
Table 1. The main data for 83317 spatial cells under consideration: $X, Y$ and $Z$ coordinates $(\mathrm{pc}), \operatorname{mode}\left(G_{\mathrm{BP}}-G_{\mathrm{RP}}\right)(\mathrm{mag}), \operatorname{mode}\left(G_{\mathrm{RP}}-W 3\right)$ (mag). The complete table is available in the Supporting Information.

\begin{tabular}{rrrrr}
\hline$X$ & $Y$ & $Z$ & $\operatorname{mode}\left(G_{\mathrm{BP}}-G_{\mathrm{RP}}\right)$ & $\operatorname{mode}\left(G_{\mathrm{RP}}-W 3\right)$ \\
\hline 0 & -1 & 165 & 1.19 & 1.53 \\
-3 & -2 & 253 & 1.20 & 1.58 \\
-4 & -1 & 179 & 1.19 & 1.58 \\
1 & -5 & 72 & 1.22 & 1.43 \\
3 & -5 & 339 & 1.20 & 1.58 \\
$\cdots$ & $\cdots$ & $\cdots$ & $\ldots$ & $\cdots$ \\
\hline
\end{tabular}

take into account a non-uniform spatial distribution of the stars, the calculated modes are referred to average positions of the stars used in the cells instead of the centres of the cells.

For each cell we calculate a distance between the average position of the stars used and the centre of the cell. A small ratio of this distance to the cell radius may be due to a random deviation of the stars from their uniform spatial distribution. A large ratio may mean a systematic loss of stars in a cell at the periphery of the space cylinder. This may lead to a bias in the related results. Therefore, we estimate the maximal acceptable ratio due to random reasons as a function of $|Z|$ by a Monte-Carlo simulation based on the spatial distribution of the stars. We use a million realizations of the simulated stars. The desired ratio appears about 0.2 for $|Z|<700 \mathrm{pc}$, increasing as $0.1 / \exp (-|Z|)$ for higher $|Z|$, where $Z$ is in $\mathrm{kpc}$, due to a lower spatial density of the stars. We use these limits to cut out the biased cells. The modes of the colours for the remaining 83317 cells are the main data for our further analysis. These data are presented in Table 1 available online.

\subsection{Longitudinal variations}

Fig. 1 shows the modes of the colours calculated in the 83317 cells as functions of the longitude $l$ for the cylinders of $\left(X^{2}+Y^{2}\right)^{0.5}<700$ and $\left(X^{2}+Y^{2}\right)^{0.5}<500 \mathrm{pc}$. The pattern in all the plots is similar and well known. For example, it can be compared with figure 6 of Gontcharov (2009) obtained for a different stellar class, OB-stars. This pattern draws four distinct regions (longitudes ranges) with a higher reddening $\operatorname{mode}\left(G_{\mathrm{BP}}-G_{\mathrm{RP}}\right)>1.35$ and $\operatorname{mode}\left(G_{\mathrm{RP}}-\right.$ $W 3)>1.75$ at $l \approx 5^{\circ}, 120^{\circ}, 175^{\circ}$, and $300^{\circ}$. The fifth region at $l \approx 30^{\circ}$ is less distinct.

For a better understanding of the pattern, Fig. 2 shows mode $\left(G_{\mathrm{RP}}-W 3\right)$ as a function of $l$ for two ranges of latitude: (a) $|b|<5^{\circ}$ and (b) $18^{\circ}<|b|<30^{\circ}$. The equatorial dust layer should dominate in the former, while the Belt layer - in the latter. Indeed, Fig. 2 shows different patterns in the different ranges of $|b|$. It attributes the three regions of a higher reddening to the equatorial and the two regions to the Belt layer. $\operatorname{mode}\left(G_{\mathrm{BP}}-G_{\mathrm{RP}}\right)$ draws a similar pattern.

The regions at $l \approx 30^{\circ}, 120^{\circ}$, and $300^{\circ}$ lie near the Galactic mid-plane and are separated from each other by $\Delta l \approx 90^{\circ}$. These regions are seen as three petals of a similar colour (i.e. similar $Z$ coordinates) in Fig. 3, where the cells with the high reddening mode $\left(G_{\mathrm{BP}}-G_{\mathrm{RP}}\right)>1.35$ are projected into the $X Y$ plane with their $Z$ coordinate shown by the colour, with its scale indicated at the right. The directions from the Sun (at the centre) to these three higher reddening petals are shown by the black lines. This pattern suggests some sinusoidal variations of the reddening in the equatorial dust layer with $l$ (with the maximal reddening at $l \approx 30^{\circ}$ ) and with $2 l$ (with the maximal reddening at $l \approx 120^{\circ}$ and $300^{\circ}$ ). The cells with mode $\left(G_{\mathrm{RP}}-W 3\right)>1.75$ show a similar pattern. The sinusoid of $l$ may be explained by the well-known gradient of the dust medium density in the directions to the centre and anticentre of the Galaxy. The sinusoid of $2 l$ may be related to the Local Arm bisecting across local space.

However, the colour of the petals at $l \approx 30^{\circ}, 120^{\circ}$, and $300^{\circ}$ in Fig. 3 is not exactly the same. This points to a deviation of the petals from the Galactic mid-plane to the north and south in the second and fourth Galactic quadrants, respectively ${ }^{6}$. This deviation is a warp of the equatorial dust layer. This warp has been known before. For example, it is seen as a higher reddening at $-25^{\circ}<b<0^{\circ}$ in the fourth quadrant and $0^{\circ}<b<+25^{\circ}$ in the second quadrant in figure 6 of Gontcharov (2010), in addition to an even higher reddening in the Gould Belt seen in the same figure at the directions to the centre and anticentre of the Galaxy.

The regions of these petals with the most deviation from the mid-plane correspond to the well-known Aquila South $\left(l \approx 33^{\circ}\right.$, $\left.b \approx-17^{\circ}\right)$, Polaris Flare $\left(l \approx 120^{\circ}, b \approx+25^{\circ}\right)$, and Chamaeleon $\left(l \approx 300^{\circ}, b \approx-17^{\circ}\right.$ ) cloud complexes (Dame et al. 2001, figure $2 \mathrm{a}$ inset). Yet, despite the deviation of these complexes from the Galactic mid-plane, the petals under consideration are dominated by the dust near the mid-plane, as seen from the colours in Fig. 3.

Finally, the 3D distribution of the cells in these three petals allows us to impose some constraints on the characteristics of the equatorial dust layer. They must be: the layer scale height $70<$ $Z_{\mathrm{A}}<250 \mathrm{pc}$, the phase $35^{\circ}<\Phi<75^{\circ}$ of the reddening variations with $\sin (l+\Phi)$ and the corresponding phase $\Phi^{\prime}=2 \Phi+90^{\circ}$ of the reddening variations with $\sin \left(2 l+\Phi^{\prime}\right)$.

The other two regions of higher reddening at $l \approx 5^{\circ}$ and $175^{\circ}$ are seen in Fig. 3 as two petals far from the Galactic midplane. Their deviation from the mid-plane, as follows from their colour in Fig. 3, corresponds to $|Z| \approx 180 \mathrm{pc}$ at $|X| \approx 500 \mathrm{pc}$ and, consequently, a tilt of $\arctan (180 / 500)=20^{\circ}$. Thus, these petals follow the known orientation of the Gould Belt. Its midplane is inclined to the Galactic mid-plane by nearly $18^{\circ}$ with the upper and lower sides directed nearly to the centre and anticentre of the Galaxy, respectively (Perryman 2009) (pp. 311-314, 324328). Consequently, these two petals of higher reddening contain the Aquila rift, Ophiuchus, Lupus (at the Belt upper side) and Taurus - Perseus - Auriga, Orion (at its lower side) cloud complexes, respectively, as seen from Dame et al. (2001, figure 2a inset).

Note that the difference of the longitudes of the high reddening petals $\Delta l=175^{\circ}-5^{\circ}=170^{\circ}$ deviates from $180^{\circ}$. This means that the centre of the Belt layer is shifted from the Sun to the second Galactic quadrant.

An approximate position of the Belt layer is drawn schematically in Fig. 3 by the ellipse.

Finally, the 3D distribution of the Belt cells allows us to put some constraints on the position, size, form and orientation of the Belt layer, assuming it is finite. It can be described as an ellipse with the semi-major axis $400 \mathrm{pc}<A<600 \mathrm{pc}$, the semi-minor axis $85 \mathrm{pc}<a<250 \mathrm{pc}$, the eccentricity $0.78<e<0.99$, and the inclination to the Galactic mid-plane $15^{\circ}<\gamma<21^{\circ}$. The Belt layer centre is shifted by $-100<x_{0}<0,+50<y_{0}<+150$, and $-50 \mathrm{pc}<z_{0}<0 \mathrm{pc}$ along the $X, Y$, and $Z$ Galactic coordinate axes,

6 The colour of the cells in the first quadrant in Fig. 3 suggests a deviation of its dust layer to the south. 

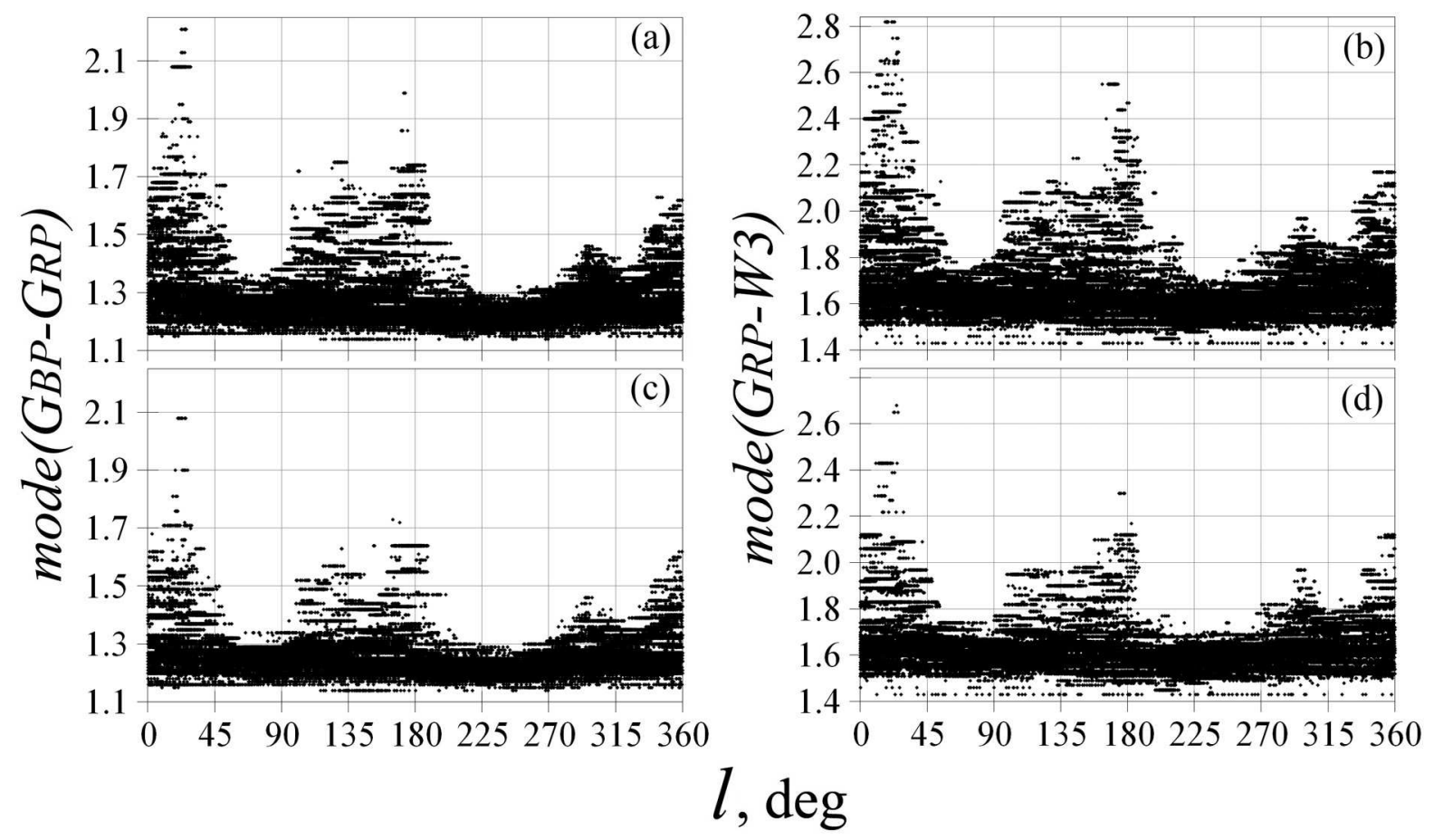

Figure 1. (a) mode $\left(G_{\mathrm{BP}}-G_{\mathrm{RP}}\right)$ and (b) mode $\left(G_{\mathrm{RP}}-W 3\right)$ as functions of $l$ for the cylinder of $\left(X^{2}+Y^{2}\right)^{0.5}<700$ pc. (c) and (d) - the same for the cylinder of $\left(X^{2}+Y^{2}\right)^{0.5}<500 \mathrm{pc}$.

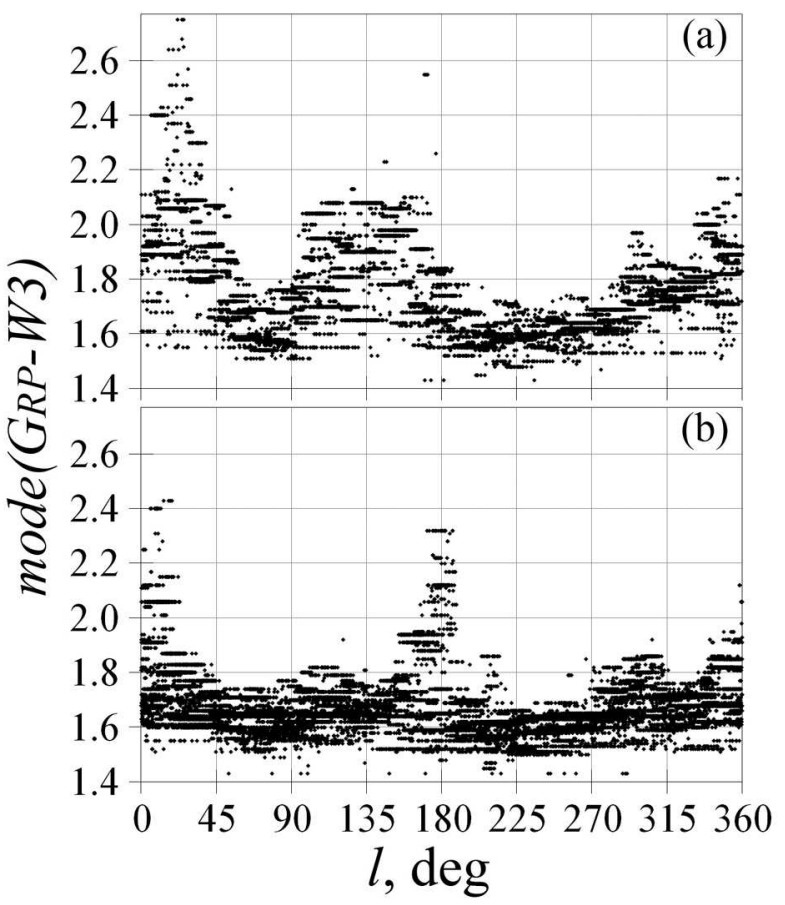

Figure 2. mode $\left(G_{\mathrm{RP}}-W 3\right)$ as a function of $l$ for (a) $|b|<5^{\circ}$ and (b) $18^{\circ}<|b|<30^{\circ}$.

respectively. The Belt layer has the scale height $50<\zeta_{\mathrm{A}}<200 \mathrm{pc}$, the angle $-20^{\circ}<\eta<+20^{\circ}$ between the semi-major axis and the direction of the maximum reddening, the angle $-20^{\circ}<\theta<$ $+20^{\circ}$ between the $Y$ Galactic coordinate axis and the line of the intersection of the mid-planes of the equatorial and Belt layers, and the phase $70^{\circ}<\phi<110^{\circ}$ of the reddening variations with $\sin (2 \lambda+\phi)$, where $\lambda$ is the longitude in the Belt mid-plane, which will be defined later.

The space with the lowest reddening, i.e. $\operatorname{mode}\left(G_{\mathrm{BP}}-G_{\mathrm{RP}}\right)<$ 1.35 , between the five petals of the higher reddening, is seen in Fig. 3 as a central white region. It is elongated in Fig. 3 from the first to the third Galactic quadrant. It is the well-known Local interstellar tunnel, or the Great tunnel (Welsh 1991; Gontcharov 2012; Gontcharov \& Mosenkov 2019). Its appearance in Fig. 3 confirms our results.

Fig. 1 (a), (b) and Fig. 2 (b) shows that the petals of the Belt are slightly asymmetric w.r.t. the Sun: the maximum reddening at $l \approx 5^{\circ}$ is higher than the one at $175^{\circ}$ for both the colours. It can be explained by the shift of the Sun w.r.t. the centre of the Belt layer.

A more pronounced asymmetry is seen for the petals of the equatorial layer, especially in Fig. 2 (a): the maximum reddening at $l \approx 120^{\circ}$ is higher than the one at $300^{\circ}$ for both the colours. It can be explained by an asymmetry of the equatorial layer around the Sun, namely, by an additional dust container in the second Galactic quadrant. Fig. 1 (d) shows that this asymmetry in the equatorial layers disappears for mode $\left(G_{\mathrm{RP}}-W 3\right)$, if we limit the space by $\left(X^{2}+Y^{2}\right)^{0.5}<500$ pc: the maxima at $120^{\circ}$ and $300^{\circ}$ becomes equal. However, this is not the case for $\operatorname{mode}\left(G_{\mathrm{BP}}-G_{\mathrm{RP}}\right)$, for which such an asymmetry persists within $\left(X^{2}+Y^{2}\right)^{0.5}<500 \mathrm{pc}$, as seen in Fig. 1 (c), and disappears only within $\left(X^{2}+Y^{2}\right)^{0.5}<400$ pc. Such different spatial variations of the colour modes within $400<$ $\left(X^{2}+Y^{2}\right)^{0.5}<500 \mathrm{pc}$ are possible, since their linear correlation coefficient in the whole space cylinder under consideration is only 0.86 . The different spatial variations of $\operatorname{mode}\left(G_{\mathrm{BP}}-G_{\mathrm{RP}}\right)$ and mode $\left(G_{\mathrm{RP}}-W 3\right)$ mean that the variations of $E\left(G_{\mathrm{BP}}-G_{\mathrm{RP}}\right)$ and $E\left(G_{\mathrm{RP}}-W 3\right)$ differ. In turn, this suggests a slightly different spatial distribution of fine and coarse dust grains, which are responsible for the former and latter reddening, respectively. 


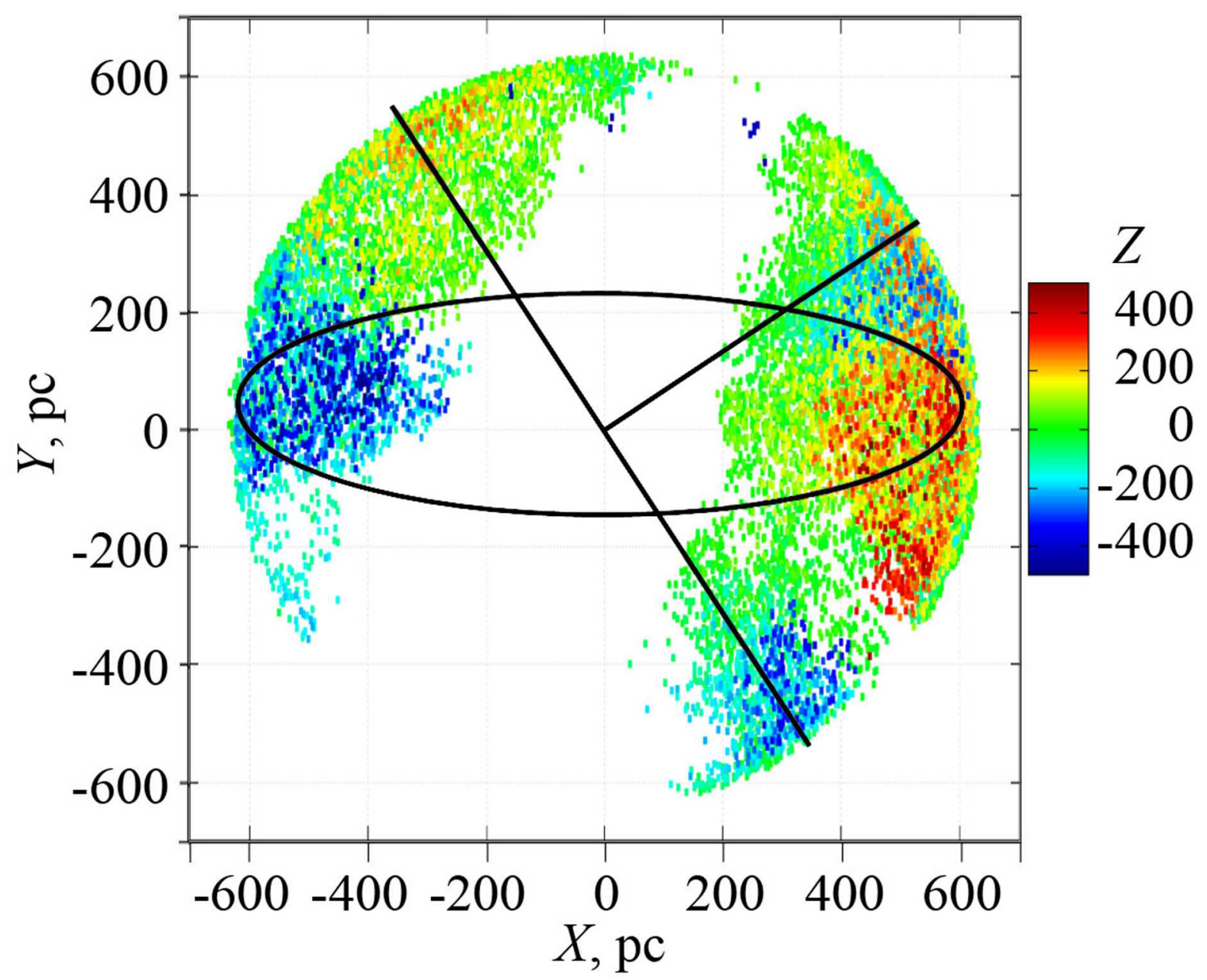

Figure 3. The cells with mode $\left(G_{\mathrm{BP}}-G_{\mathrm{RP}}\right)>1.35$ projected into the $X Y$ plane with their $Z$ coordinate shown by the colour scale at the right. The Sun is at the centre. The Galactic centre is to the right. The approximate position of the Gould Belt is shown by the black ellipse. The directions to the three regions with a higher reddening in the equatorial dust layer are shown by the black lines.

Thus, any model of the 3D dust distribution in the first kiloparsec should take into account (i) the additional dust container in the second quadrant beyond $400 \mathrm{pc}$ from the Sun and (ii) possible slightly different spatial distribution of the fine and coarse dust and related possible spatial variation of the extinction law near the Galactic mid-plane. However, GM20 presented in this study, does not take into account any warp or asymmetry of the equatorial dust layer. Moreover, up to now there is no model which takes these features into account correctly. This can potentially be a subject for future studies.

\section{MODELS}

\subsection{Simplest model}

The simplest model implies an exponential vertical distribution of dust in one layer, without any variation on $l$. Hence, the cumulative reddening of a star follows the barometric law (Parenago 1954, p. 265):

$E_{0} R\left(1-\mathrm{e}^{-\left|Z-Z_{0}\right| / Z_{\mathrm{A}}}\right) Z_{\mathrm{A}} /\left|Z-Z_{0}\right|$,

where $E_{0}$ is the differential reddening in the mid-plane of the layer, $R$ is the distance from the Sun, $Z_{0}$ is the vertical offset for the mid- plane of the dust layer with respect to the Sun, and $Z_{\mathrm{A}}$ is the scale height of the dust layer.

Also, we derive one additional parameter for each colour the linear gradient of the dereddened colour $\left(G_{\mathrm{BP}}-G_{\mathrm{RP}}\right)_{0}$ or $\left(G_{\mathrm{RP}}-W 3\right)_{0}$ with $|Z|$. Note that these colours themselves can be derived for the simplest model only with a very large uncertainty. Hence, they are fixed as 1.145 and 1.435 , respectively, following the findings of Paper I. We derive the least root-meansquare deviations of this model from the data in the 83317 cells with the following parameters: $Z_{0}=-6 \mathrm{pc}$ for $\operatorname{mode}\left(G_{\mathrm{BP}}-G_{\mathrm{RP}}\right)$ versus $-1 \mathrm{pc}$ for mode $\left(G_{\mathrm{RP}}-W 3\right), Z_{\mathrm{A}}=170$ versus $180 \mathrm{pc}$, $E_{0}=0.4$ versus 0.5 mag $k p c^{-1}, \Delta\left(G_{\mathrm{BP}}-G_{\mathrm{RP}}\right)_{0}=-0.024|Z|$ versus $\Delta\left(G_{\mathrm{RP}}-W 3\right)_{0}=-0.018|Z|$, where $Z$ is in kpc. We note the rather large scale heights as compared to estimates in the literature (Perryman 2009, pp. 470-471, 496-497). Yet, they are rather diverse: from 35 (Vergely et al. 1998) and $<70$ (Jurić et al. 2008) to 140 (Robin et al. 2003) and 188 pc (Drimmel \& Spergel 2001).

The derived reddenings across the whole dust half-layer below or above the Sun are:

$E\left(G_{\mathrm{BP}}-G_{\mathrm{RP}}\right)=0.068$,

$E\left(G_{\mathrm{RP}}-W 3\right)=0.090$. 
By use of the extinction law of WC19 the values (8) and (9) correspond to $E(B-V)=0.051$ and $0.052 \mathrm{mag}$, respectively. These estimates will be compared with others in Sect. 5 .

The corresponding colour excess ratio (CER) is $E\left(G_{\mathrm{RP}}-\right.$ $W 3) / E\left(G_{\mathrm{BP}}-G_{\mathrm{RP}}\right)=1.32$. It fits good with the CERs 1.24 , 1.28 , and 1.33 provided by the extinction laws of DIB14 for the high latitudes, SMS16, and WC19, respectively. However, our CER disagrees with the CER 1.69 from the law of CCM89 with $R_{\mathrm{V}}=3.1$.

\subsection{Model GM20}

Here we apply the model GM20 to the data in order to derive a set of its parameters together with the additional parameters - the modes of the dereddened colour $\left(G_{\mathrm{BP}}-G_{\mathrm{RP}}\right)_{0}$ or $\left(G_{\mathrm{RP}}-W 3\right)_{0}$ and their linear gradients with $|Z|$.

GM20 implies the existence of two dust layers with their midplanes intersecting at an angle $\gamma$ : the equatorial layer and the layer in the Gould Belt, with the scale heights $Z_{\mathrm{A}}$ and $\zeta_{\mathrm{A}}$, respectively. The line of the intersection of the mid-planes of these layers is rotated from the $Y$ axis by the angle $\theta$. The scheme of the mid-planes of the layers is shown in Fig. 4. Note that we do not consider any warp of the layers.

Expanding far beyond the space under consideration, the equatorial layer is considered as infinite along the $X$ and $Y$ coordinates. The equatorial layer mid-plane is shifted w.r.t. the Sun by $Z_{0}$ along the $Z$ coordinate.

In contrast, the Gould Belt layer mid-plane is limited by the ellipse with the semi-major axis $A$, semi-minor axis $a$, eccentricity $e$, and angle $\eta$ between the semi-major axis and the direction of the maximum reddening. The centre of this ellipse is shifted w.r.t. the Sun by $x_{0}, y_{0}$, and $z_{0}$ along $X, Y$, and $Z$, respectively. This is a difference of GM20 from its previous versions, where the Belt mid-plane was limited by a circle shifted w.r.t. the Sun only along the direction perpendicular to the Belt mid-plane.

Another difference of GM20 from its previous versions follows from the findings in Sect. 2.1: the longitudinal variations of reddening in the equatorial layer include two terms, with $\sin (l)$ and $\sin (2 l)$. Therefore, the cumulative reddening $E\left(G_{\mathrm{BP}}-G_{\mathrm{RP}}\right)$ or $E\left(G_{\mathrm{RP}}-W 3\right)$ is the sum of three terms. They follow a barometric law and vary along the mid-plane of the corresponding layer:

$[E+S \sin (l+\Phi)] R\left(1-\mathrm{e}^{-\left|Z-Z_{0}\right| / Z_{\mathrm{A}}}\right) Z_{\mathrm{A}} /\left|Z-Z_{0}\right|$,

$\left[D \sin \left(2 l+2 \Phi+90^{\circ}\right)\right] R\left(1-\mathrm{e}^{-\left|Z-Z_{0}\right| / Z_{\mathrm{A}}}\right) Z_{\mathrm{A}} /\left|Z-Z_{0}\right|$

for the single and double sinusoidal longitudinal variations in the equatorial layer, respectively, and

$\left[G_{0}+G_{1} \sin (2 \lambda+\phi)\right] \min \left(R, R_{0}\right)\left(1-\mathrm{e}^{-|\zeta| / \zeta_{\mathrm{A}}}\right) \zeta_{\mathrm{A}} /|\zeta|$

for the variations in the Belt layer. $E$ and $G_{0}$ are the constant terms, $S, D$, and $G_{1}$ are the amplitudes, and $\Phi, 2 \Phi+90^{\circ}$ and $\phi$ are the phases. The coordinates of a star/point/cell in the coordinate system of the Belt are the latitude $\beta$ counted from the Belt mid-plane, the longitude $\lambda$ counted from the direction of the maximal reddening, and the distance from the mid-plane of the Belt $\zeta . R_{0}$ is the distance from the Sun to the Belt layer edge at the line of sight of a star under consideration. The term $\min \left(R, R_{0}\right)$ in equation (12) allows us to calculate the reddening in the Belt layer to its edge or to the star, whichever is closer. These quantities are calculated as:

$R_{0}=a /\left(1-[\mathrm{e} \cos (\lambda-\eta)]^{2}\right)^{0.5}$, $\sin (\beta)=\cos (\gamma) \sin (b)-\sin (\gamma) \cos (b) \cos (l)$,

$\tan (\lambda-\theta)=\cos (b) \sin (l) /[\sin (\gamma) \sin (b)+\cos (\gamma) \cos (b) \cos (l)]$,

$\zeta=\min \left(R, R_{0}\right) \sin (\beta)$

We assume that $Z_{0}$ and $Z_{\mathrm{A}}$ are the same for the terms of the equatorial layer. This assumption is based on our analysis of Fig. 3 and its analogues in the $X Z$ and $Y Z$ planes. This assumption wiil be fully validated in our future work, where these parameters will be made free.

\subsection{GM20 results}

The solution, obtained for the 20 free parameters of GM20, is presented in Table 2 (only two quantities among $A, a$ and $e$ are independent). This solution corresponds to the least root-meansquare deviation of GM20 from the data $\left[\operatorname{mode}\left(G_{\mathrm{BP}}-G_{\mathrm{RP}}\right)\right.$ and mode $\left(G_{\mathrm{RP}}-W 3\right)$ in the 83317 cells]. This solution was achieved through a consideration of several trillions sets of the parameters varied within the constraints, listed in Table 2, and the direct calculation of the residuals and their root-mean-square deviation.

Since one star is used for the mode calculation in several cells, the data in the cells are not independent. To check the robustness of the solution and to estimate the uncertainties of the derived model parameters, we apply a resampling method. We fill our cylinder space by 122 spherical cells, positioned so that they adjoin but do not intersect each other. The size of each cell is defined by the $Z$ coordinate of its centre following relation (6). For example, the mid-plane layer cells have a radius of $110 \mathrm{pc}$. The set of the cells does not fill the whole space cylinder. We shift the set as a whole by $25 \mathrm{pc}$ steps within $-50<X<50$ or $-50<Y<50$ or by 10 pc steps within $-10<Z<10$ pc to produce $5 \times 5 \times 3=75$ samples of the cells inside the space cylinder. This allows us to use almost all the stars of the cylinder. For each sample we calculate the colour modes for the central coordinates of each cell by use of all the stars in that cell. Since the cells do not intersect each other, the mode values are independent of each other. These modes are used to obtain a solution, with 20 parameters of our model, giving the least root-mean-square deviation of the model from the data. Thus, one sample of the cells gives us one realization of the resampling method and, consequently, one set of the derived parameters. The final distribution of each parameter in the 75 realizations/solutions appears to be nearly Gaussian. The average values of the parameters are close to those for 83317 cells in Table 2. The standard deviations of the parameters in 75 realizations/solutions are presented in Table 2 as the uncertainties of the parameters.

The solutions for the two colours are the same within the uncertainties, except their $Z_{\mathrm{A}}$, and, naturally, dereddened colours. Yet, the difference of $2 \sigma$ between $Z_{\mathrm{A}}$ for two colours is not significant. It is worth noting that all the derived angles agree with the position of the five petals of higher reddening in Fig. 3.

The reddening maxima of the Belt layer in Fig. 1, 2 and 3 appear nearly at the directions to the Galactic centre and anticentre. The eccentricity (0.97) of the Belt layer mid-plane is rather large. The differences $G_{0}-G_{1}$ is rather moderate. All these facts mean that our model successfully reproduces a significant fraction of the 


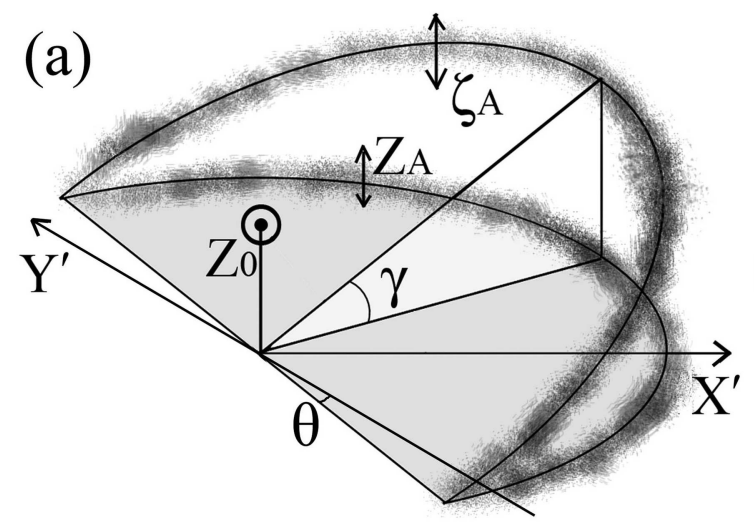

(b)

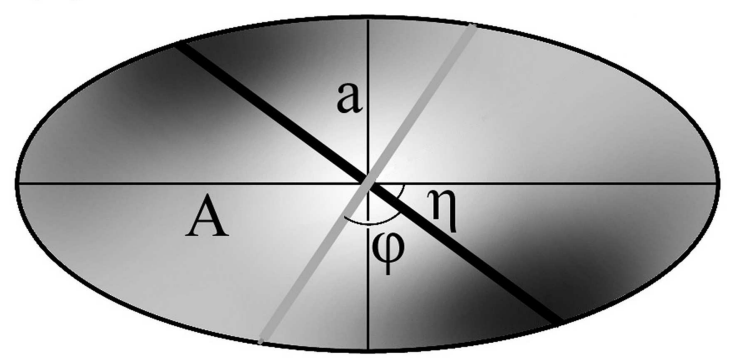

Figure 4. The scheme of the mid-planes of the Galactic dust layers near the Sun: (a) for the two intersecting mid-planes, (b) for the elliptical mid-plane of the Gould Belt. $X^{\prime}$ and $Y^{\prime}$ are the projections of the Galactic coordinate axes $X$ and $Y$ into the mid-plane of the equatorial dust layer.

Table 2. The parameters of the Galactic dust layer and the giant clump in GM20.

\begin{tabular}{lccc}
\hline Parameter & Constraints & $G_{\mathrm{BP}}-G_{\mathrm{RP}}$ & $G_{\mathrm{RP}}-W 3$ \\
\hline Dereddened colour $(\mathrm{mag})$ & $-1.18 / 1.41-1.52$ & $1.14 \pm 0.01$ & $1.44 \pm 0.01$ \\
Colour gradient along $|Z|\left(\mathrm{mag} \mathrm{kpc}^{-1}\right)$ & $-0.08-0$ & $-0.022 \pm 0.010$ & $-0.015 \pm 0.010$ \\
$\gamma\left(^{\circ}\right)$ & $15-21$ & $17 \pm 2$ & $17 \pm 2$ \\
$\theta\left(^{\circ}\right)$ & $-20-+20$ & $-3 \pm 4$ & $-4 \pm 4$ \\
$\eta\left(^{\circ}\right)$ & $-20-+20$ & $2 \pm 4$ & $0 \pm 4$ \\
$Z_{0}(\mathrm{pc})$ & $-30-+30$ & $-7 \pm 10$ & $-10 \pm 10$ \\
$x_{0}(\mathrm{pc})$ & $-100-0$ & $-25 \pm 15$ & $-5 \pm 15$ \\
$y_{0}(\mathrm{pc})$ & $+50-+150$ & $+135 \pm 15$ & $+115 \pm 15$ \\
$z_{0}(\mathrm{pc})$ & $-50-0$ & $-26 \pm 15$ & $-30 \pm 15$ \\
$A(\mathrm{pc})$ & $400-650$ & $600 \pm 50$ & $600 \pm 50$ \\
$a$ & & $146 \pm 37$ & $146 \pm 37$ \\
$e$ & $0.78-0.99$ & $0.97 \pm 0.01$ & $0.97 \pm 0.01$ \\
$Z_{A}\left(\mathrm{pc}^{\circ}\right)$ & $70-250$ & $150 \pm 15$ & $180 \pm 15$ \\
$\zeta_{A}(\mathrm{pc})$ & $50-200$ & $55 \pm 15$ & $65 \pm 15$ \\
$E\left(\mathrm{mag} \mathrm{kpc}^{-1}\right)$ & $<1$ & $0.35 \pm 0.07$ & $0.46 \pm 0.10$ \\
$S\left(\mathrm{mag} \mathrm{kpc}{ }^{-1}\right)$ & $<1$ & $0.04 \pm 0.07$ & $0.04 \pm 0.10$ \\
$\Phi\left(^{\circ}\right)$ & $35-75$ & $39 \pm 10$ & $39 \pm 10$ \\
$\left.D(\mathrm{mag} \mathrm{kpc})^{-1}\right)$ & $<1$ & $0.16 \pm 0.07$ & $0.20 \pm 0.10$ \\
$G 0\left(\mathrm{mag} \mathrm{kpc}^{-1}\right)$ & $<1$ & $0.45 \pm 0.07$ & $0.50 \pm 0.10$ \\
$G 1\left(\mathrm{mag} \mathrm{kpc}^{-1}\right)$ & $<1$ & $0.22 \pm 0.07$ & $0.24 \pm 0.10$ \\
$\phi\left(^{\circ}\right)$ & $70-110$ & $98 \pm 10$ & $98 \pm 10$ \\
\hline
\end{tabular}

Belt dust, which is contained in two, nearly opposite, regions at $-30^{\circ}<l<+30^{\circ},+5^{\circ}<b<+25^{\circ}$ and $150^{\circ}<l<220^{\circ}$, $-25^{\circ}<b<-5^{\circ}$. As pointed out in Sect. 2.1, these regions include the Aquila rift, Ophiuchus, Lupus and Taurus - Perseus - Auriga, Orion cloud complexes, respectively.

The spatial distribution of the Belt dust can differ from the distribution of its gas, stellar clusters and associations. However, Table 2 shows the position of the Belt layer centre w.r.t. the Sun (average $X=-15, Y=+125, Z=-28, R=129 \mathrm{pc}$ ) near the position (albeit rather uncertain) derived earlier from the distribution of young stars (Bobylev 2014), see also Perryman (2009, p. 325).

The scale heights $Z_{\mathrm{A}}$ and $\zeta_{\mathrm{A}}$ of the equatorial and Belt layers, respectively, show the ratio of $2.7-2.8$ in agreement with the note from Perryman (2009, p. 325) 'the Gould Belt is three times as compressed vertically as the Galactic belt, each shows the same increasing concentration for stars, interstellar dust, and stellar groups'.

Our solution gives rather large scale heights $Z_{\mathrm{A}}$, which are comparable with those obtained for the simplest model and those of Robin et al. (2003) and Drimmel \& Spergel (2001) mentioned in Sect. 3.1. Regardless, our solution is preferable as it is based on a sample covering a wider range of $Z$ and, for the first time, definitely covering the entire depth of the dust layer (or layers). Do note that such large scale heights are ultimately defined by the fact that the colour modes of our sample still increase (become redder) at a high $|Z|$ (say, $450 \mathrm{pc}$ ). Some previous studies could not show a reddening behavior at such a high $|Z|$ due to poor or no data. Some examples of such studies are Vergely et al. (1998) with their very low estimate of the dust layer scale height ( $35 \mathrm{pc}$ ) and the $3 \mathrm{D}$ reddening maps 
of LVV14 and Lallement et al. (2019, hereafter LVV19) ${ }^{7}$. It is also worth noting that a rather thick dust layer near the Sun in GM20 agrees with the scale height estimates (always $>120 \mathrm{pc}$ ) of edge-on galaxies from De Geyter et al. (2014) and Mosenkov et al. (2018), see their tables 3 and 4, respectively.

GM20 gives reddenings across the whole dust half-layer below or above the Sun:

$E\left(G_{\mathrm{BP}}-G_{\mathrm{RP}}\right)=0.073$,

$E\left(G_{\mathrm{RP}}-W 3\right)=0.105$,

with nearly the same contribution of the equatorial and Belt layers: 0.035 versus 0.038 and 0.055 versus 0.050 mag to the former and latter reddening, respectively. These estimates will be compared with others in Sect. 5.

Estimates (17) and (18) give the CER 1.44, which fits well with the CERs 1.24, 1.28, and 1.33, provided by the extinction laws of DIB14 for the high latitudes, SMS16, and WC19, respectively, while fits slightly worse with 1.69 from the law of CCM89 with $R_{\mathrm{V}}=3.1$.

\subsection{HR diagrams and isochrones}

All 101810 giants of our sample are shown in Fig. 5 with the HR diagrams before and after correcting for the reddenings $E\left(G_{\mathrm{BP}}-G_{\mathrm{RP}}\right)$ and $E\left(G_{\mathrm{RP}}-W 3\right)$ and extinctions $A_{\mathrm{G}}$ and $A_{\mathrm{W} 3}$. The reddenings are derived in the current study with GM20, while the extinctions are calculated from the reddenings by use of the extinction law of WC19. It gives $A_{\mathrm{G}_{\mathrm{RP}}}=1.429 E\left(G_{\mathrm{BP}}-G_{\mathrm{RP}}\right)$ and $A_{\mathrm{W} 3}=0.073 E\left(G_{\mathrm{RP}}-W 3\right)$.

Note that the extinction law is important in Fig. 5 only for the ordinates. Therefore, one should pay more attention to the distributions along the abscissas as our direct results.

For the median $|Z|$ of our sample (212 pc), Paper I gives the median age $3 \mathrm{Gyr}$ and $[\mathrm{Fe} / \mathrm{H}]=-0.11$. These estimates seem to be reasonable being in good agreement with the estimates from Girardi (2016). We show the corresponding PARSEC ${ }^{8}, \mathrm{MIST}^{9}$ and IAC-BaSTI ${ }^{10}$ isochrones in Fig. 5 against the background of the distribution for the sample. The clump median absolute magnitudes and intrinsic colours correspond to the bottom of the leftmost $U$ shape loop of the isochrones. The rightmost nearly vertical lines of the isochrones are the giant branch.

Comparing the original diagrams in plots (a) and (b) with the diagrams corrected for the reddening and extinction in plots (c) and (d), one can see that GM20 significantly reduces the scatter of the sample and suggests proper corrections. Namely, the leftmost $\mathrm{U}$ shape loops of the isochrones fit the distribution tops within a few hundredths of a magnitude for the PARSEC, MIST and IACBaSTI isochrones in plot (c) and for the PARSEC isochrone in plot (d). Our findings in Paper I suggest that MIST is less reliable in its predictions for $W 3$, probably, due to an issue in its colour$T_{\text {eff }}$ relation and/or bolometric correction used. A deviation of the

\footnotetext{
7 http://stilism.obspm.fr

8 PARSEC version $1.2 \mathrm{~S}$ assuming $[\alpha / \mathrm{Fe}]=0$, solar metallicity $Z=$ 0.0152 , mass-loss efficiency $\eta=0.2$, where $\eta$ is the free parameter in the Reimers' law (Reimers 1975).

9 MIST version 1.2 assuming $[\alpha / \mathrm{Fe}]=0$ and the reference protosolar metallicity $Z=0.0142$.

10 IAC-BaSTI version assuming $[\alpha / \mathrm{Fe}]=0$, overshooting, diffusion, mass-loss efficiency $\eta=0.3$.
}

MIST isochrone from the distribution top in plot (d) agrees with this suggestion.

Fig. 5 shows the distribution tops in perfect agreement with estimates (2)-(5) for the clump modes from Paper I.

The distribution of the sample in Fig. 5 confirms that the branch and asymptotic branch giants are only a minor admixture to the clump giants, which does not affect the main modes of the distribution. However, an admixture of the branch giants is noticeable as an excess of the stars just below the bulk in Fig. 5 (c) and (d), i.e. for

$\left(G_{\mathrm{BP}}-G_{\mathrm{RP}}\right)_{0} \approx 1.2$

and $M_{\mathrm{G}_{\mathrm{RP}}} \approx 0.1$ or

$\left(G_{\mathrm{RP}}-W 3\right)_{0} \approx 1.5$

and $M_{\mathrm{W} 3}>-1.5 \mathrm{mag}$. It is seen that in plots (c) and (d) all the theoretical giant branches perfectly fit the observed giant branches, except the MIST giant branch in ' $\left(G_{\mathrm{RP}}-W 3\right)_{0}$ versus $M_{\mathrm{W} 3}$ '. In contrast, this excess of the branch giants is seen in plots (a) and (b) at $G_{\mathrm{BP}}-G_{\mathrm{RP}} \approx 1.3$ and $\left(G_{\mathrm{RP}}-W 3\right) \approx 1.66$. The noticeable differences between these estimates and those (19) and (20) point out that GM20 accurately takes into account the considerable reddening of the sample.

The estimates from GM20:

$\left(G_{\mathrm{BP}}-G_{\mathrm{RP}}\right)_{0}=(1.14 \pm 0.01)-(0.022 \pm 0.010)|Z|$,

$\left(G_{\mathrm{RP}}-W 3\right)_{0}=(1.44 \pm 0.01)-(0.015 \pm 0.010)|Z|$,

where $Z$ is in kpc. They agree with estimates (4) and (5) obtained in Paper I.

\section{DATA VERSUS MODELS AND MAPS}

In Fig. 6 we compare observed mode $\left(G_{\mathrm{BP}}-G_{\mathrm{RP}}\right)$ with predictions from the most reliable $3 \mathrm{D}$ reddening models and maps ${ }^{11}$ for all 83317 cells. mode $\left(G_{\mathrm{RP}}-\right.$ W3 $)$ shows similar results. To convert the calculated reddenings into the colours, we use the extinction law of WC19 and our estimates (21) and (22).

Any reasonable change of the extinction law has little, if any, effect on this conversion. The same is true for any reasonable change of the coefficients before $|Z|(|Z|$ gradients) in estimates (21) and (22), since these coefficients are small. With most stars of the sample within $|Z|<400 \mathrm{pc}$, any reasonable change of these coefficients lead to a change of the dereddened colours (21) and (22) of $<0.01$ mag. Therefore, the only values, which can affect the comparison and, particularly, Fig. 6, are the dereddened colours (21) and (22) at $Z=0$. Any change of them leads to an overall offset of the model/map predictions, which would be seen as an overall vertical shift of the points in Fig. 6.

It is seen that such a vertical shift would give a better fit to the data for the predictions of DCL03 ${ }^{12}$, LVV19, and Green et al. $\left(2019\right.$, hereafter GSF19) ${ }^{13}$, i.e. would put the most amount of their points closer to the one-to-one relation shown by the black bisector line in Fig. 6. More precisely, the best fit of their predictions to the data suggests $\left(G_{\mathrm{BP}}-G_{\mathrm{RP}}\right)_{0}=1.17,1.18$ and 1.17;

11 Some of these maps and models have been briefly described and compared with other data by Gontcharov \& Mosenkov (2017b, 2018).

12 The DCL03 estimates are calculated by use of the code of Bovy et al. (2016), https://github.com/jobovy/mwdust

13 http://argonaut.skymaps.info/ 

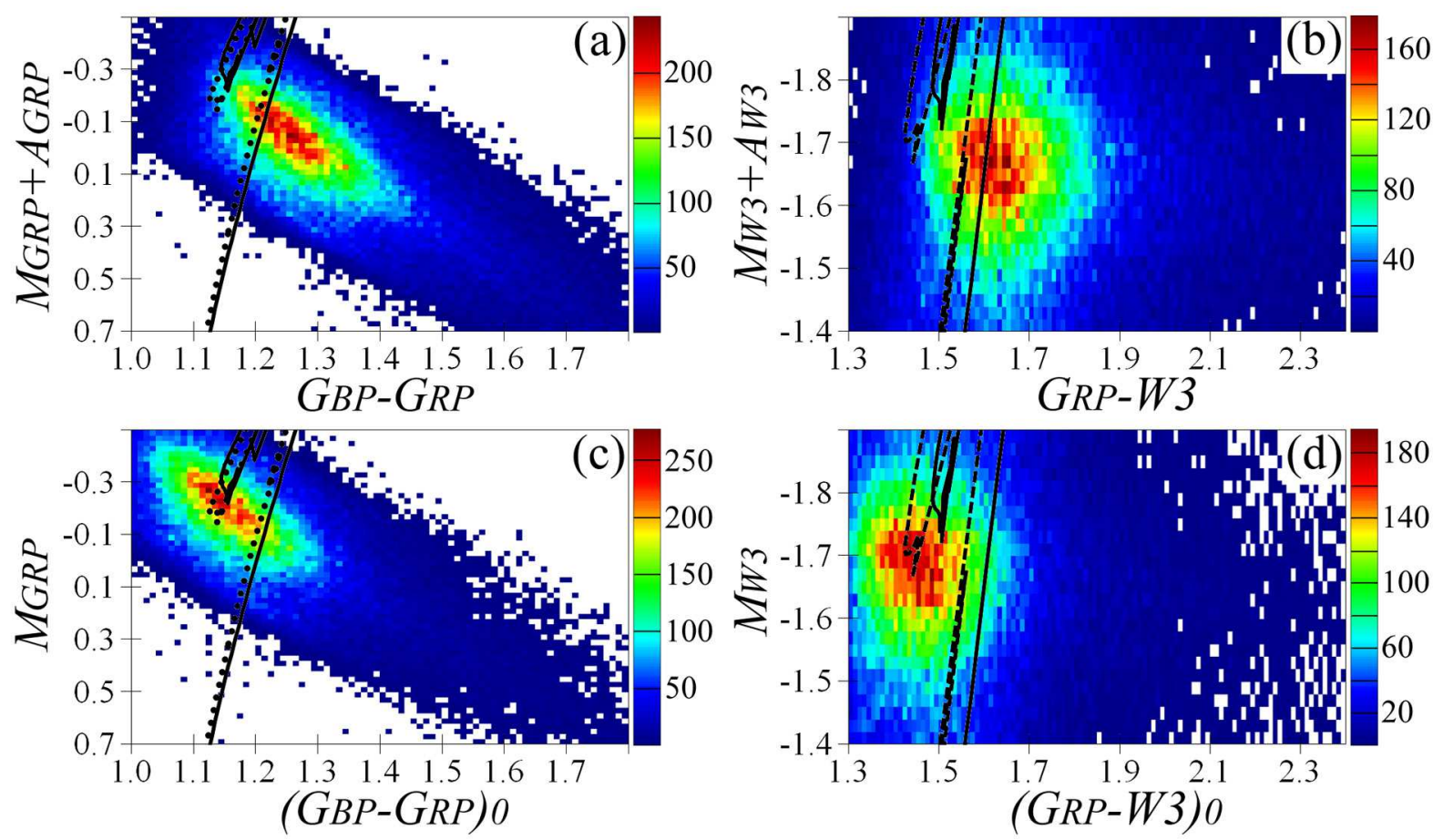

Figure 5. The diagrams (a) ' $G_{\mathrm{BP}}-G_{\mathrm{RP}}$ versus $M_{\mathrm{G}}+A_{\mathrm{G}_{\mathrm{RP}}}$ ', (b) ' $G_{\mathrm{RP}}-W 3$ versus $M_{\mathrm{W} 3}+A_{\mathrm{W} 3}$ ', (c) ' $\left(G_{\mathrm{BP}}-G_{\mathrm{RP}}\right)_{0}$ versus $M_{\mathrm{G}}$ ', and (d) '( $\left.G_{\mathrm{RP}}-W 3\right)_{0}$ versus $M_{\mathrm{W} 3}$ ' for the 101810 stars of our sample before and after the correction for the reddenings from GM20 in combination with the extinction law of WC19. The bins are 0.01 and 0.02 mag for the abscissas and ordinates, respectively. The number of the stars in each bin is shown by the colour scale on the right. The PARSEC, MIST, and IAC-BaSTI isochrones for $3 \mathrm{Gyr}$ and $[\mathrm{Fe} / \mathrm{H}]=-0.11$ are shown by the dashed, solid and dotted black curves, respectively. Since the PARSEC and MIST isochrones coincide with each other on the scale of plots (a) and (c), the PARSEC isochrone is not shown in these plots. The IAC-BaSTI presents no isochrone for plots (b) and (d).

$\left(G_{\mathrm{RP}}-W 3\right)_{0}=1.50,1.51$ and 1.50 at $Z=0$, respectively. However, these values are far from all the isochrone predictions in Fig. 5, except the MIST isochrone for ' $\left(G_{\mathrm{RP}}-W 3\right)_{0}$ versus $M_{\mathrm{W} 3}$ '. Yet, the latter seems to be less reliable, as noted above. Moreover, such high estimates of the colours cannot explain the existence of some cells with $\operatorname{mode}\left(G_{\mathrm{BP}}-G_{\mathrm{RP}}\right)<1.17$ and $\operatorname{mode}\left(G_{\mathrm{RP}}-W 3\right)<1.50$ seen, for example, in Fig. 1. This may mean a systematic underestimation of the reddening by DCL03, LVV19, and GSF19. Such an underestimation, if real, introduces an especially large systematic uncertainty into low reddenings, i.e. for nearby and high-latitude stars.

It is seen in Fig. 6 that the model of Arenou, Grenon \& Gomez (1992, hereafter AGG92) slightly overestimates high and underestimates low reddenings. This underestimation is explained by the constraint applied by AGG92: $A_{\mathrm{V}}<0.1 \mathrm{mag}$ for $|b| \geq 60^{\circ}$.

Also Fig. 6 shows that GM20, G12, G17 and the simplest model from Sect. 3.1 drop the most cells near the bisector. Consequently, these maps and models agree with estimates (21) and (22) (for GM20 - by definition).

Such predictions by a perfect map/model should cover exactly the full range of mode $\left(G_{\mathrm{BP}}-G_{\mathrm{RP}}\right)$ in Fig. 6 with a minimal scatter around the bisector line. It is worth noting that both the proper coverage of the range and the scatter are independent from estimates (21) and (22). The $|Z|$ gradients of the intrinsic colours in estimates (4), (5), (21), and (22) are negative. This makes distant stars bluer and having no effect on the nearby stars. Therefore, such a $|Z|$ gradient makes the predicted colour range narrower and does not shift the whole bulk of the points, i.e. does not change an offset. Any lowering of the reddening also makes the predicted colour range narrower. But no map/model needs to make its range narrower, as seen from Fig. 6. Moreover, we need wider colour ranges for the simplest model and DCL03.

A rather moderate fit of the simplest model is achieved by the adjustment of its parameters in Sect. 3.1. The imperfection of this model confirms that the only dust layer without longitudinal variations, in any case, is not enough to describe the real 3D distribution of dust.

Fig. 6 shows that GM20 is better than G12. However, GM20 underestimates the highest reddenings, i.e. for $R>400 \mathrm{pc}$, because it does not take into account the asymmetry of the equatorial layer, which was discussed in Sect. 2.1.

Also Fig. 6 shows that G17, LVV19, and GSF19 give nearly equally good predictions, if one pays no attention to the overall offsets, which depend on accepted estimates (21) and (22).

An increase of $\left(G_{\mathrm{BP}}-G_{\mathrm{RP}}\right)_{0}$ and $\left(G_{\mathrm{RP}}-W 3\right)_{0}$ at $Z=0$ w.r.t. estimates (21) and (22) can improve the overall offsets of DCL03, LVV19, and GSF19 from the data. However, it cannot change the linear correlation coefficients between these predictions and the data. Therefore, these correlation coefficients are useful to check, which prediction is better among those with a minimal scatter around the bisector line and with a proper coverage of the colour range. Table 3 with the correlation coefficients shows that, as expected from Fig. 6, G17, LVV19, and GSF19 are better than the remaining ones and, finally, G17 and GM20 are the best among the maps and models, respectively.

Table 3 shows that, generally, the maps tend to give better predictions than the models do. The reason is that the maps and models describe the dust medium by tables and formulas, respectively. The former can take into account some short-term fluctuations of the medium, while the latter draw only a smooth pattern of the 


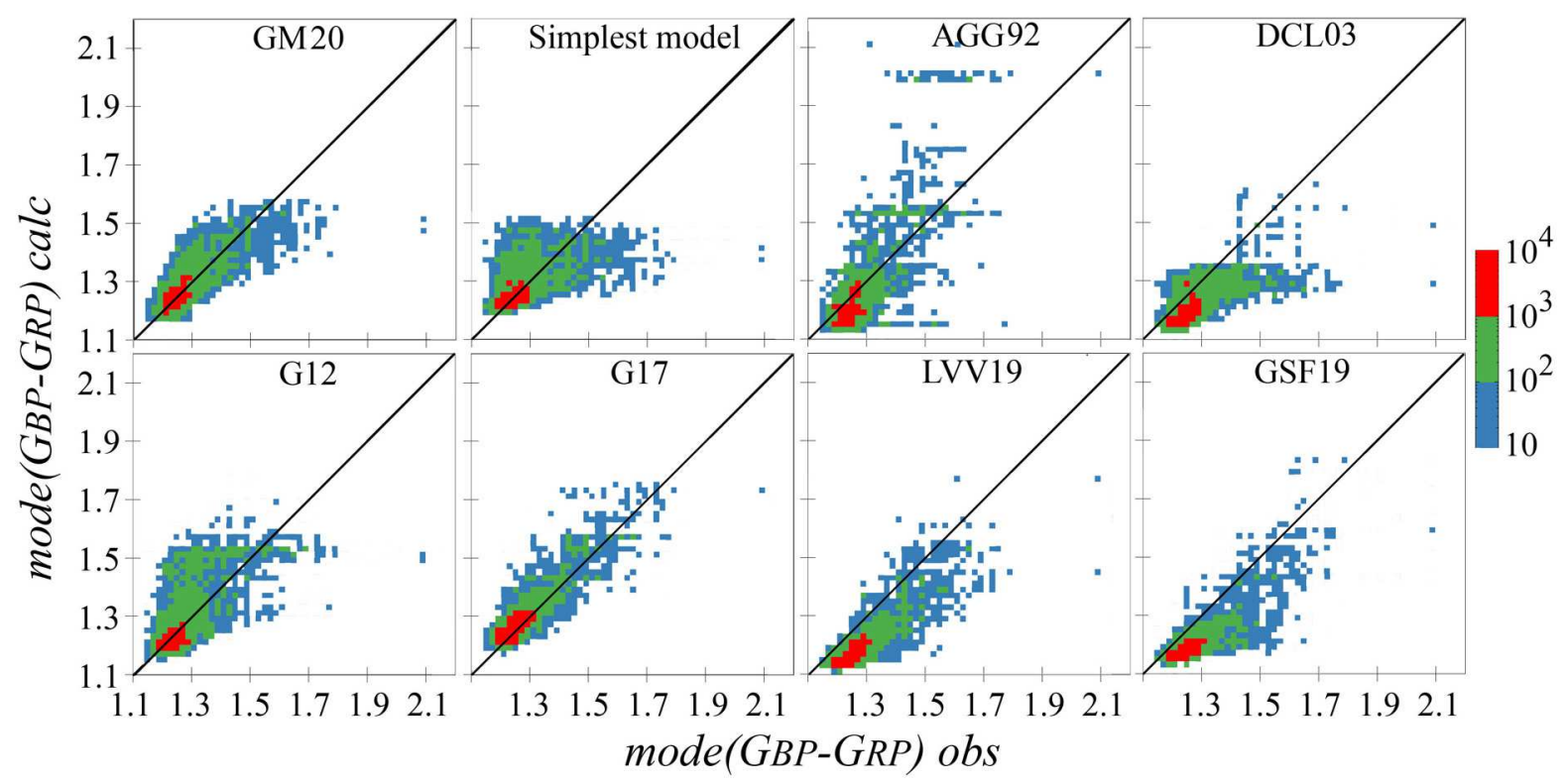

Figure 6. The relation between the observed and calculated mode $\left(G_{\mathrm{BP}}-G_{\mathrm{RP}}\right)$ for various models and maps. The number of the stars in each bin of $0.02 \times 0.02$ mag is shown by the colour scale on the right.

Table 3. The correlation coefficients between the data and predictions from the models and maps.

\begin{tabular}{lcc}
\hline Model / map & $\operatorname{mode}\left(G_{\mathrm{BP}}-G_{\mathrm{RP}}\right)$ & $\operatorname{mode}\left(G_{\mathrm{RP}}-W 3\right)$ \\
\hline Simplest model & 0.52 & 0.54 \\
Model AGG92 & 0.76 & 0.75 \\
Model DCL03 & 0.66 & 0.67 \\
Model G12 & 0.68 & 0.68 \\
Model GM20 & 0.78 & 0.79 \\
Map G17 & 0.87 & 0.86 \\
Map LVV19 & 0.86 & 0.83 \\
Map GSF19 & 0.82 & 0.81 \\
\hline
\end{tabular}

medium. However, in contrast to maps, models allow us to derive the key parameters of the medium, as well as their relations and consequences.

\subsection{Comparison over the sky}

The reddenings calculated from $\operatorname{mode}\left(G_{\mathrm{BP}}-G_{\mathrm{RP}}\right)$ and mode $\left(G_{\mathrm{RP}}-W 3\right)$ for 83317 cells by use of relations (21) and (22) are compared over the sky with the reddening or extinction estimates from various models and maps in Fig. 7. All these reddening/extinction estimates are presented with the same scale based on the extinction law of WC19.

The upper row of the plots in Fig. 7 shows the data, the second row shows the corresponding reddenings from GM20, then we show the predictions from three maps and three models in the left and right columns, respectively.

We arrange cells with similar $l$ and $b$ in Fig. 7 to show the nearby ones as background, while more distant ones as foreground. We select the size of the points in Fig. 7 small enough in order to eliminate any obscuration of them at the middle and high latitudes. Some obscuration exists only when the volume density of the cells is the highest, i.e. near the Galactic mid-plane, within $|b|<15^{\circ}$. However, even at $|b|<15^{\circ}$ the uniform distribution of the stars and cells along $X$ and $Y$ leads to a dominance of the cells with a higher reddening, while the nearby cells with a lower reddening are rare and, hence, not important.

Fig. 7 reveals the same five regions of a higher reddening/extinction as in Fig. 1, 2, and 3. They are circled in the plot for the observed mode $\left(G_{\mathrm{BP}}-G_{\mathrm{RP}}\right)$. These regions are drawn almost equally precise and detailed by the observed modes, G17, LVV19, and GSF19 (the region at $l \approx 300^{\circ}$ is not considered by GSF19), but not by DCL03.

The reason of this fail of DCL03 is evident from Fig. 7. It describes the dust distribution by a warped exponential disc with the addition of the local Orion arm features at $l \approx 80^{\circ}$ and $260^{\circ}$. However, a warp of the highly reddened regions of the disc, seen for DCL03 in Fig. 7, is actually the Belt layer tilted to the Galactic mid-plane. The real warp of the equatorial dust layer, as shown in Sect. 2.1, appears in the second and fourth Galactic quadrants at $R>400 \mathrm{pc}$. Moreover, the regions with a higher reddening at $l \approx 80^{\circ}$ and $260^{\circ}$, drawn by DCL03 in Fig. 7 , are absent in the data within $700 \mathrm{pc}$ from the Sun. These features were included into the DCL03 model following the features seen in some 2D dust emission maps at an unknown distance. Probably they are related to some distant parts of the local arm, outside the space under consideration.

Fig. 7 shows that, again, the maps give a more detailed and realistic picture than the models.

Note that AGG92 shows some pixelization of the sky following the coordinate partitioning during the creation of this model.

Also, it is seen that GM20 draws a pattern closer to the data than the previous version G12 does. 


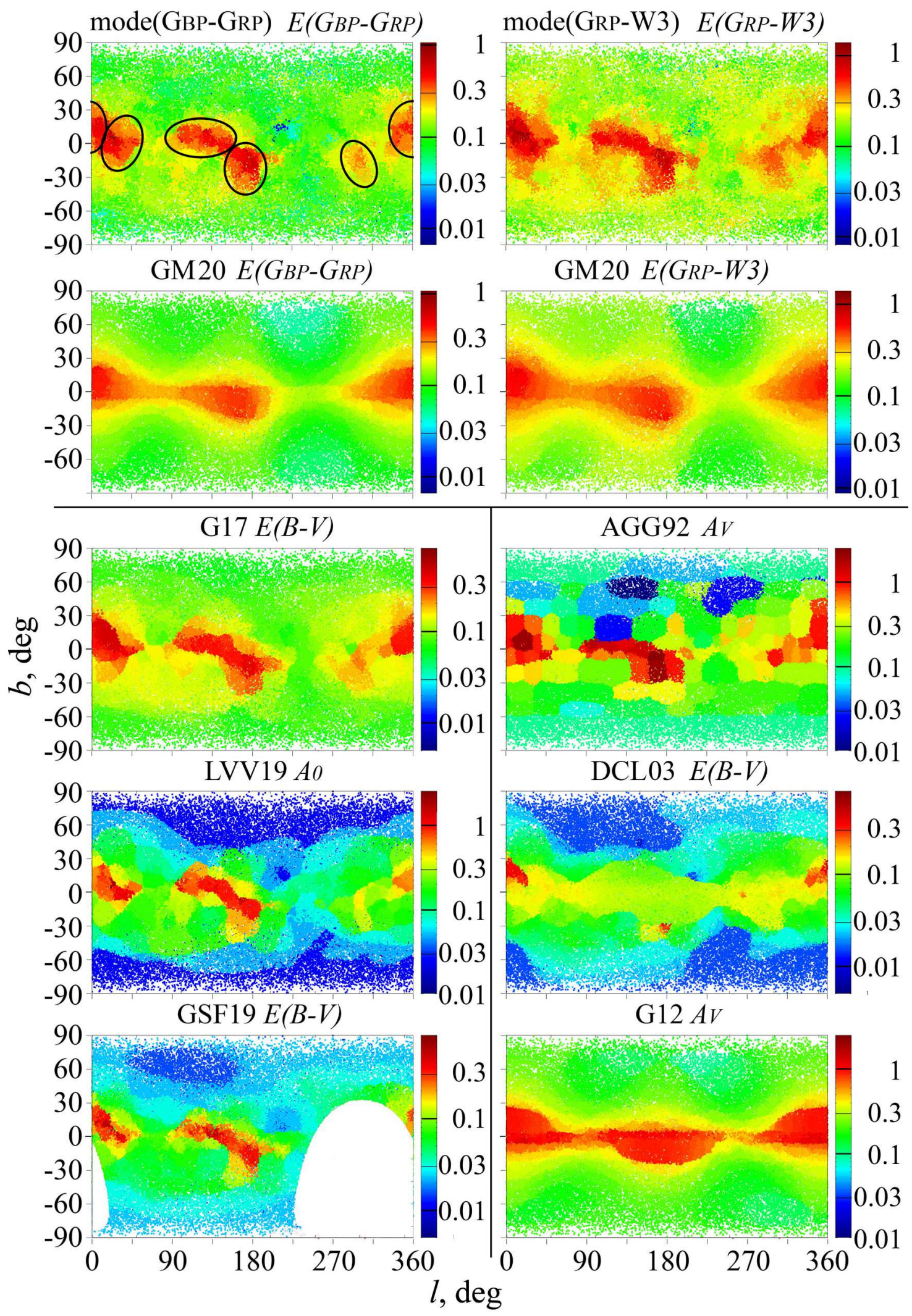

Figure 7. The variations of the reddening/extinction from mode $\left(G_{\mathrm{BP}}-G_{\mathrm{RP}}\right)$, mode $\left(G_{\mathrm{RP}}-W 3\right)$ and various models and maps for 83317 cells over the sky in Galactic coordinates. The five regions of a higher reddening are circled in the plot for the observed $\operatorname{mode}\left(G_{\mathrm{BP}}-G_{\mathrm{RP}}\right)$. 


\section{REDDENING ACROSS THE WHOLE DUST LAYER}

Different estimates of the reddening across the whole dust layer are discussed in Paper I. Here we make some additional notes on this subject.

Fig. 7 shows that the data, models and maps give different representation of the reddening/extinction at the middle and high latitudes.

In particular, in the upper row plots of Fig. 7 the yellow colour for $E\left(G_{\mathrm{RP}}-W 3\right)$ instead of the green colour for $E\left(G_{\mathrm{BP}}-G_{\mathrm{RP}}\right)$ dominates at the middle and high latitudes. This means that the observed $E\left(G_{\mathrm{RP}}-W 3\right)$ exceeds $E\left(G_{\mathrm{BP}}-G_{\mathrm{RP}}\right)$, when both the reddenings are derived from the data and normalized with the same extinction law of WC19. This difference is not due to an offset. Hence, it cannot be changed by a change of dereddened colour (21) or (22). Instead, this difference is due to a wider range of the normalized $E\left(G_{\mathrm{RP}}-W 3\right)$ w.r.t. the normalized $E\left(G_{\mathrm{BP}}-G_{\mathrm{RP}}\right)$ at the middle and high latitudes. These ranges can be the same, if we use another extinction law. Thus, this points to a slight deviation of the empirical extinction law from that of WC19 at the middle and high latitudes ${ }^{14}$. The same effect is seen for the reddening estimates from GM20 (the second row of the plots in Fig. 7). The difference between the GM20 plots in Fig. 7 corresponds to the difference between $Z_{A}$ for $G_{\mathrm{BP}}-G_{\mathrm{RP}}$ and $G_{\mathrm{RP}}-W 3$ in Table 2 . All these findings may suggest a slightly different vertical spatial distribution of the fine and coarse dust grains.

The colour of the middle and high latitudes in Fig. 7 allows us to rank the maps and models in ascending order of their estimates of low extinctions/reddenings as: LVV19, DCL03, GSF19, and AGG92 - show an apparent underestimation of the low extinctions/reddenings w.r.t. the observed ones in the upper row plots versus G12, GM20, and G17, which agree with each other and with the observed values. This diversity of the estimates of low reddening, including the reddening across the whole dust layer, is noted in Paper I.

We compare in Fig. 8 several 3D reddening models and maps in their ability to predict the observed mode $\left(G_{\mathrm{BP}}-G_{\mathrm{RP}}\right)$ for the 2788 cells within $\left(X^{2}+Y^{2}\right)^{0.5}<120$ pc, i.e. within a vertical cylinder across the whole dust layer. This cylinder is quite narrow to contain as unreddened nearby stars, as the stars behind the whole dust layer. A similar figure can be shown for $\operatorname{mode}\left(G_{\mathrm{RP}}-W 3\right)$.

The vertical offsets of AGG92, DCL03, LVV19, and GSF19 from the bisector line in Fig. 8 can be corrected by accepting an intrinsic colour higher than (21). However, as noted in Sect. 4, this cannot correct any prediction of the colour range of the observed mode $\left(G_{\mathrm{BP}}-G_{\mathrm{RP}}\right)$, i.e. the accordance of the abscissa and ordinate ranges in Fig. 8. The abscissa range reflects an observed diversity of the colours due to the reddening across the whole dust layer. Hence, the best predictions should cover the same colour range along the ordinate. However, Fig. 8 shows that the simplest model, DCL03, LVV19, and GSF19 cover too narrow colour ranges (i.e. their ranges along the ordinate). The only way to make their colour ranges wider is a higher reddening. Thus, Fig. 8 confirms that the simplest model, DCL03, LVV19, and GSF19 underestimate the reddening across the whole dust layer. The same is true for a similar figure of mode $\left(G_{\mathrm{RP}}-W 3\right)$.

Some models and maps show rather tight correlations of their reddenings between each other within the whole space cylinder

14 The extinction laws of DIB14 for the high latitudes, WC19, SMS16, and CCM89 with $R_{\mathrm{V}}=3.1$ give a similar slight deviation from the empirical law at the middle and high latitudes. under consideration [the correlation coefficients for mode $\left(G_{\mathrm{BP}}-\right.$ $\left.G_{\mathrm{RP}}\right)$ and $\operatorname{mode}\left(G_{\mathrm{RP}}-W 3\right)$ are averaged]:

- 0.91 for GM20 versus G12,

- 0.86 for G17 versus LVV19,

- 0.84 for LVV19 versus GSF19,

- 0.83 for GM20 versus G17,

- 0.82 for G17 versus GSF19.

Similar tight correlations have been found by Gontcharov \& Mosenkov (2019) between G17, G12, AGG92, on the one hand, and a previous (albeit rather similar) version LVV14 of LVV19, on the other hand. This means that, at least, the independent reddening estimates from G17, LVV19, and GSF19 can be basically described as some linear combinations of each other (this is also evident from Fig. 6). In particular, Gontcharov \& Mosenkov (2019) have shown in their figure 11 and their equation (4) that G17 differs from LVV14 mostly by the constant offset of $\Delta E(B-V)=0.06 \mathrm{mag}$. This offset can be interpreted as the difference between the estimates of the reddening across the whole dust half-layer from G17 and LVV14. Indeed, a similar offset of $\Delta E\left(G_{\mathrm{BP}}-G_{\mathrm{RP}}\right)=0.07 \mathrm{mag}[\Delta E(B-V)=0.05$ mag with the extinction law of WC19] between the predictions of G17 and LVV19 is seen in Fig. 8. Such a constant offset means that the difference between the maps/models is more noticeable for low reddenings, i.e. near the Sun and at high latitudes. This offset also means that, if $\mathrm{G} 17$ overestimates $E\left(G_{\mathrm{BP}}-G_{\mathrm{RP}}\right)$ by $0.01 \mathrm{mag}$, then LVV19 underestimates it by $0.06 \mathrm{mag}$ [by $\Delta E(B-V)=0.045 \mathrm{mag}$ with the extinction law of WC19].

Table 4 summarizes the comparable estimates of the reddening across the whole dust half-layer below or above the Sun from Paper I and the current paper. They agree with each other. The estimates from Paper I and GM20 converge to the average value $E(B-V)=$ $0.059 \pm 0.003$ mag by use of the extinction law of WC19. Note that these new estimates are lower than that from the previous version of the model, G12, also presented in Table 4. However, the diversity of the estimates from different maps and models, summarized in table 6 of Paper I, shows that the estimation of the reddening across the whole dust half-layer is still an issue.

\section{CONCLUSIONS}

In this study we exploited the complete sample of 101810 Gaia DR2 giants. It has been selected in Paper I within the clump domain of the Hertzsprung-Russell diagram and in the space cylinder with the radius $700 \mathrm{pc}$ around the Sun, up to $|Z|<1800 \mathrm{pc}$ along the $Z$ Galactic coordinate. Owing to the accurate parallaxes and photometry in the $G_{\mathrm{BP}}$ and $G_{\mathrm{RP}}$ bands from Gaia DR2, in combination with the WISE photometry in the $W 3$ band, this sample is the first one, which is complete for the space across the whole Galactic dust layer near the Sun.

In the current paper, we presented GM20, the revised version of the 3D dust distribution model introduced by Gontcharov in 2009. We used GM20 to explain the spatial variations of the colour modes mode $\left(G_{\mathrm{BP}}-G_{\mathrm{RP}}\right)$ and mode $\left(G_{\mathrm{RP}}-W 3\right)$. This allowed us to derive simultaneously the key properties of the Galactic dust layer, the intrinsic colours and absolute magnitudes of the giant clump, and vertical gradients of the intrinsic colours with $|Z|$.

We revealed five regions with high reddening $\operatorname{mode}\left(G_{\mathrm{BP}}-\right.$ $\left.G_{\mathrm{RP}}\right)>1.35$ and mode $\left(G_{\mathrm{RP}}-W 3\right)>1.75$. The regions at $l \approx 30^{\circ}, 120^{\circ}$, and $300^{\circ}$ contain the Aquils South, Polaris Flare, and Chamaeleon cloud complexes, respectively, and belong to the 


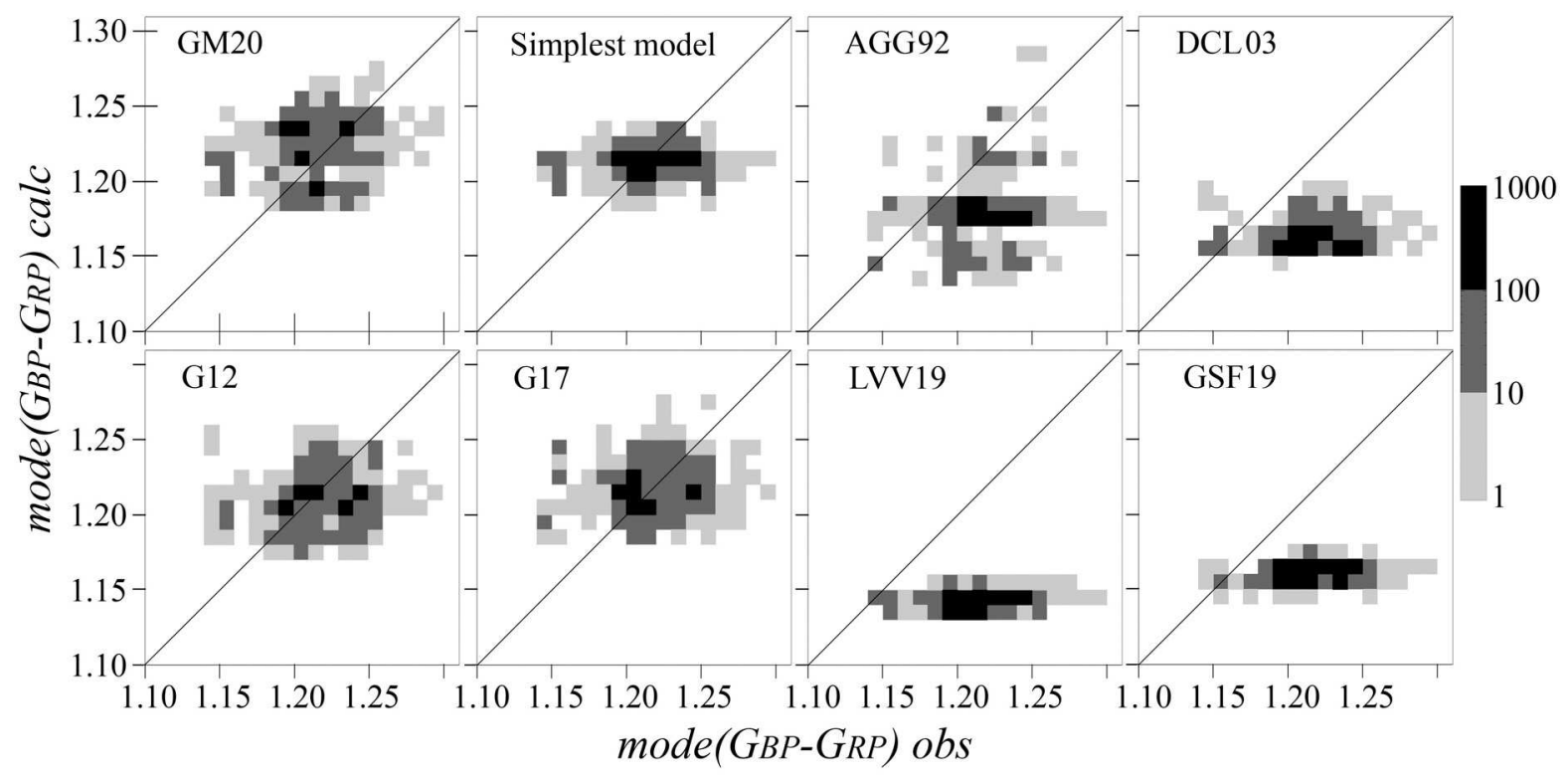

Figure 8. The same as Fig. 6 but for 2788 cells within $\left(X^{2}+Y^{2}\right)^{0.5}<120$ pc. The number of the stars in each bin of $0.01 \times 0.01$ mag is shown by the colour scale on the right.

Table 4. Some estimates of the extinction or reddening across the whole dust half-layer below or above the Sun.

\begin{tabular}{|c|c|c|}
\hline Source & Original estimate & $E(B-V)$ by WC19 \\
\hline Paper I, narrow cylinder & $E\left(G_{\mathrm{BP}}-G_{\mathrm{RP}}\right)=0.080$ & 0.061 \\
\hline This study, simplest model & $E\left(G_{\mathrm{BP}}-G_{\mathrm{RP}}\right)=0.068$ & 0.051 \\
\hline This study, model GM20 & $E\left(G_{\mathrm{BP}}-G_{\mathrm{RP}}\right)=0.073$ & 0.055 \\
\hline Paper I, narrow cylinder & $E\left(G_{\mathrm{RP}}-W 3\right)=0.100$ & 0.058 \\
\hline This study, simplest model & $E\left(G_{\mathrm{RP}}-W 3\right)=0.090$ & 0.052 \\
\hline This study, model GM20 & $E\left(G_{\mathrm{RP}}-W 3\right)=0.105$ & 0.061 \\
\hline Model G12 & $A_{\mathrm{V}}=0.214$ & 0.068 \\
\hline
\end{tabular}

equatorial dust layer. Other ones at $l \approx 5^{\circ}$ and $175^{\circ}$ contain Aquila rift, Ophiuchus, Lupus and Taurus - Perseus - Auriga, Orion cloud complexes, respectively, and belong to the dust layer of the Gould Belt inclined to the Galactic mid-plane at about $17^{\circ}$.

Taking into account this analysis, we proposed for GM20 the $3 \mathrm{D}$ dust distribution in two intersecting dust layers, with exponential vertical and sinusoidal longitudinal variations of the dust spatial density in each layer. Twenty free parameters of our GM20 model were calculated for the least root-mean-square deviation of this model from the data. mode $\left(G_{\mathrm{BP}}-G_{\mathrm{RP}}\right)$ and mode $\left(G_{\mathrm{RP}}-W 3\right)$ gave similar solutions, but slightly different equatorial layer scale heights of $150 \pm 15$ and $180 \pm 15 \mathrm{pc}$, respectively. The current solution for GM20 presents the Belt layer as an ellipse, oriented nearly between the centre and anticentre of the Galaxy, with a maximum reddening in roughly these directions, and with a semi-major and -minor axes of 600 and $146 \mathrm{pc}$, respectively. This agrees well with the known structure of the Belt. Note that the Belt dust container produces a considerable extinction at the middle and high latitudes.

GM20 estimates for nearby clump giants $\left(G_{\mathrm{BP}}-G_{\mathrm{RP}}\right)_{0}=$ $(1.14 \pm 0.01)-(0.022 \pm 0.010)|Z|$ and $\left(G_{\mathrm{RP}}-W 3\right)_{0}=(1.44 \pm$ $0.01)-(0.015 \pm 0.010)|Z|$, where $Z$ is expressed in $\mathrm{kpc}$, agree with the estimates from Paper I, obtained in a different approach.
Also, GM20 estimates agree with the PARSEC, MIST and IAC-BaSTI theoretical isochrones for the clump giants, with their expected median age of $3 \mathrm{Gyr}$ and median metallicity of $[\mathrm{Fe} / \mathrm{H}]=$ -0.11 . The only exception is the MIST isochrone based on the MIST predictions for $W 3$. It deviates, probably, due to an issue in its colour $-T_{\text {eff }}$ relation and/or bolometric correction used.

We compared the observed mode $\left(G_{\mathrm{BP}}-G_{\mathrm{RP}}\right)$ and mode $\left(G_{\mathrm{RP}}-W 3\right)$ with the predictions from GM20 and several $3 \mathrm{D}$ reddening models and maps. GM20 and G17 appeared to be the best 3D reddening model and map, respectively.

All our results from Paper I and from this study agree with the reddening across the whole dust half-layer below or above the Sun $E(B-V)=0.059 \pm 0.003 \mathrm{mag}$.

DCL03, LVV19, and GSF19 need much higher giant clump intrinsic colours to reduce their residuals with the data. Such colours cannot explain as much data with the lower observed dereddened colours. Thus, we are inclined to conclude that DCL03, LVV19, and GSF19 systematically underestimate low reddenings. This is confirmed by the too narrow range of their predictions for the colours inside the vertical space cylinder across the whole dust layer.

However, G17, LVV19, and GSF19 show such high linear correlation coefficients in the whole space under consideration that 
their reddening predictions differ almost exclusively by constant offsets. These offsets might arise from their different estimates for the low reddenings near the Sun and across the whole dust halflayer. Thus, a correct estimation of such low reddenings, as well as the creation of the samples of unreddened stars, seem to be the key points for the modern investigations of extinction and reddening in the Galaxy.

\section{ACKNOWLEDGEMENTS}

We thank the reviewer, Dr. Xiaodian Chen for useful comments. We thank Jeremy Mutter for assistance with English syntax. We thank Anton Dryanichkin, Evgeny Evseev, Marina Garanina, Evgeny Gordeev, Pavel Popov, and Natalia Svetlova for their help in calculations.

We acknowledge financial support from the Russian Science Foundation (grant no. 20-72-10052).

The research described in this paper makes use of Filtergraph (Burger et al. 2013), an online data visualization tool developed at Vanderbilt University through the Vanderbilt Initiative in Data-intensive Astrophysics (VIDA) and the Frist Center for Autism and Innovation (FCAI, https://filtergraph.com). The resources of the Centre de Données astronomiques de Strasbourg, Strasbourg, France (http://cds.u-strasbg.fr), including the SIMBAD data base and the X-Match service, were widely used in this study. This work has made use of data from the European Space Agency (ESA) mission Gaia (https://www.cosmos.esa.int/gaia), processed by the Gaia Data Processing and Analysis Consortium (DPAC, https://www. cosmos.esa.int/web/gaia/dpac/consortium). This publication makes use of data products from the Wide-field Infrared Survey Explorer, which is a joint project of the University of California, Los Angeles, and the Jet Propulsion Laboratory/California Institute of Technology.

\section{DATA AVAILABILITY}

The data underlying this article are available in the article and in its online supplementary material.

\section{REFERENCES}

Arenou F., Grenon M., Gomez A., 1992, A\&A, 258, 104 (AGG92)

Bobylev V. V., 2014, Astrophysics, 57, 583

Bovy J., Rix H.-W., Green G. M., Schlafly E. F., D.P. Finkbeiner, 2016, ApJ, 818,130

Burger D., Stassun K. G., Pepper J., Siverd R. J., Paegert M., De Lee N. M., Robinson W. H., 2013, Astronomy and Computing, 2, 40

Cardelli J. A., Clayton G. C., Mathis J. S., 1989, ApJ, 345, 245 (CCM89)

Choi J., Dotter A., Conroy C., Cantiello M., Paxton B., Johnson B. D., 2016, ApJ, 823, 102

Dame T. M., Hartmann Dap, Thaddeus P., 2001, ApJ, 547, 792

Davenport J. R. A. et al., 2014, MNRAS, 440, 3430 (DIB14)

De Geyter G., Baes M., Camps P., Fritz J., De Looze I., Hughes T. M., Viaene S., Gentile G., 2014, MNRAS, 441, 869

Dotter A., 2016, ApJS, 222, 8

Drimmel R., Spergel D. N., 2001, ApJ, 556, 181

Drimmel R., Cabrera-Lavers A., López-Corredoira M., 2003, A\&A, 409, 205 (DCL03)

Gaia Collaboration, 2018a, A\&A, 616, A1

Gaia Collaboration, 2018b, A\&A, 616, A4
Girardi L., 2016, ARA\&A, 54, 95

Gontcharov G. A., 2009, Astron. Lett., 35, 780 (G09)

Gontcharov G. A., 2010, Astron. Lett., 36, 584

Gontcharov G. A., 2012, Astron. Lett., 38, 87 (G12)

Gontcharov G. A., 2017a, Astron. Lett., 43, 472 (G17)

Gontcharov G. A., 2017b, Astron. Lett., 43, 545

Gontcharov G. A., Mosenkov A. V., 2017a, MNRAS, 470, L97

Gontcharov G. A., Mosenkov A. V., 2017b, MNRAS, 472, 3805

Gontcharov G. A., Mosenkov A. V., 2018, MNRAS, 475, 1121

Gontcharov G. A., Mosenkov A. V., 2019, MNRAS, 483, 299

Gontcharov G. A., Mosenkov A. V., 2020, arXiv:2011.11113 (Paper I)

Green G. M., Schlafly E., Zucker C., Speagle J. S., Finkbeiner D., 2019, ApJ, 887, 93 (GSF19)

Hidalgo S. L. et al., 2018, ApJ, 856, 125

Jurić M. et al., 2008, ApJ, 673, 864

Lallement R., Vergely J. L., Valette B., Puspitarini L., Eyer L., Casagrande L., 2014, A\&A, 561, A91 (LVV14)

Lallement R., Babusiaux C., Vergely J. L., Katz D., Arenou F., Valette B., Hottier C., Capitanio L., 2019, A\&A, 625, A135 (LVV19)

Marigo P. et al., 2017, ApJ, 835, 77

Meisner A. M., Finkbeiner D. P., 2015, ApJ, 798, 88 (MF15)

Mosenkov A. V. et al., 2018, A\&A, 616, A120

Önal Taş Ö., Bilir S., Seabroke G. M., Karaali S., Ak S., Ak T., Bostanci Z. F., 2016, Publications of the Astronomical Society of Australia, 33, 44

Parenago P. P., 1954, A Course in Stellar Astronomy [in Russian]. GITTL, Moscow

Paxton B., Bildsten L., Dotter A., Herwig F., Lesaffre P., Timmes F., 2011, ApJS, 192, 3

Paxton B. et al., 2013, ApJS, 208, 4

Perryman M., 2009, Astronomical Applications of Astrometry: Ten Years of Exploitation of the Hipparcos Satellite Data. Cambridge Univ. Press, Cambridge, UK

Reimers D., 1975, Mem. Soc. R. Sci. Liege, 8, 369

Robin A. C., Reylé C., Derrière S., Picaud S., 2003, A\&A, 409, 523

Schlegel D. J., Finkbeiner D. P., Davis M., 1998, ApJ, 500, 525 (SFD98)

Schlafly E. F. et al., 2016, ApJ, 821, 78 (SMS16)

Vergely J.-L., Freire Ferrero R., Egret D., Köppen J., 1998, A\&A, 340, 543

Wang S., Chen X., 2019, ApJ, 877, 116 (WC19)

Welsh B., 1991, ApJ, 373, 556

Wright E. L. et al., 2010, AJ, 140, 1868

This paper has been typeset from a $\mathrm{T}_{\mathrm{E}} \mathrm{X} / \mathrm{LAT} \mathrm{EX}$ file prepared by the author. 